\title{
Formation of intrathermocline eddies at ocean fronts by wind-driven destruction of potential vorticity
}

\author{
Leif N. Thomas * \\ Department of Physical Oceanography, Woods Hole Oceanographic Institution, \\ Woods Hole, Massachusetts
}

\begin{abstract}
A mechanism for the generation of intrathermocline eddies (ITEs) at wind-forced fronts is examined using a high resolution numerical simulation. Favorable conditions for ITE formation result at fronts forced by "down-front" winds, i.e. winds blowing in the direction of the frontal jet. Down-front winds exert frictional forces that reduce the potential vorticity (PV) within the surface boundary in the frontal outcrop, providing a source for the low-PV water that is the materia prima of ITEs. Meandering of the front drives vertical motions that subduct the low-PV water into the pycnocline, pooling it into the coherent anticyclonic vortex of a submesoscale ITE. As the fluid is subducted along the outcropping frontal isopycnal, the low-PV water, which at the surface is associated with strongly baroclinic flow, re-expresses itself as water with nearly zero absolute vorticity. This generation of strong anticyclonic vorticity results from the tilting of the horizontal vorticity of the frontal jet, not from vortex squashing. During the formation of the ITE, high-PV water from the pycnocline is upwelled alongside the subducting low-PV surface water. The positive correlation between the ITE's velocity and PV fields results in an upward,
\end{abstract}


along-isopycnal eddy PV flux that scales with the surface frictional PV flux driven by the wind. The relationship between the eddy and wind-induced frictional PV flux is nonlocal in time, as the eddy PV flux persists long after the wind forcing is shut off. The ITE's PV flux affects the large-scale flow by driving an eddy-induced transport or bolus velocity down the outcropping isopycnal layer with a magnitude that scales with the Ekman velocity.

Key words: fronts, eddies, potential vorticity, subduction

\section{Introduction}

Lenses of well mixed water with anomalous thermohaline properties are a common feature in the stratified interior of the world ocean (Kostianoy and Belkin, 1989). Such features have been referred to as intrathermocline eddies (ITEs) (Dugan et al., 1982) or submesoscale coherent vortices (SCVs) (McWilliams, 1985). The distinguishing characteristics of ITEs are: a central core with anomalously weak stratification and anticyclonic vorticity, a convex (concave) shape to the upper (lower) isopycnal surface that bounds the stratification anomaly, an interior maximum in the velocity field, an azimuthallysymmetric, coherent structure, and a small horizontal width relative to the first baroclinic Rossby radius of deformation (McWilliams, 1985). The combination of the ITEs' weak stratification and anticyclonic vorticity is a signature of the low values of potential vorticity (PV) found within their cores. In fact, it is the low-PV anomaly of the ITE that is its defining feature, since the velocity

* Present address: Department of Environmental Earth System Science, Stanford University, 473 Via Ortega, Stanford, CA 94305.

Email address: leift@stanford.edu (Leif N. Thomas ). 
and density field can be reconstructed (assuming that the flow is primarily balanced) from the PV anomaly alone using a PV inversion technique (Hoskins et al., 1985). Therefore, ITE formation relies on the creation of low-PV water masses and it also depends on the PV anomaly being spatially isolated. Hence, as argued by McWilliams (1985), realistic SCV/ITE generation mechanisms must satisfy two conditions: they must provide a source of low PV and do this in a spatially intermittent fashion. Several SCV/ITE generation mechanisms that match these conditions are described below.

McWilliams (1985) described a SCV formation mechanism involving patchy diapycnal mixing events of small spatial scale and subsequent spin-up of anticyclonic circulation via geostrophic adjustment. In this mechanism, diabatic processes change the PV of the fluid. PV can also be modified by friction. Indeed, D'Asaro (1988) outlined a process by which frictional torques arising from bottom drag acting on a boundary current could drive SCV formation. In this mechanism, the concentration of frictionally-induced low PV water into coherent structures typical of SCVs was attributed to flow separation triggered by variations in bottom topography. Spall (1995) showed, using numerical experiments, that SCVs can also be formed at meandering upper-ocean fronts with an initial cross-front PV gradient characterized by low PV on the dense side of the front. In this mechanism, SCVs are generated as meander-driven vertical circulation subducts low-PV water along the frontal outcrop into the stratified interior. During subduction, an anticyclonic circulation is induced owing to PV conservation and vortex squashing.

In this paper, a mechanism for ITE formation at wind-forced upper ocean fronts will be described in which PV non-conservation and three-dimensional frontal dynamics play critical roles. At such fronts, the process of wind-driven 
frictional destruction of PV of Thomas (2005) is active and provides a source for the low-PV ITE core water. To illustrate this mechanism, the paper will include an overview of PV dynamics and the role of atmospheric forcing in changing the PV at upper ocean fronts and a detailed analysis of a high resolution numerical experiment in which ITE formation at a wind-forced front was simulated.

\section{Potential vorticity dynamics}

\subsection{The potential vorticity equation}

Changes in the PV, which in this paper is defined to be the full Ertel PV

$$
q=\omega_{a} \cdot \nabla b
$$

where $\boldsymbol{\omega}_{a}=f \hat{k}+\nabla \times \mathbf{u}$ is the absolute vorticity, $f$ is the Coriolis parameter, and $b=-g \rho / \rho_{o}$ is the buoyancy, result from convergences/divergences of the PV flux, i.e.

$$
\frac{\partial q}{\partial t}=-\nabla \cdot \mathbf{J}
$$

where the PV flux

$$
\mathbf{J}=q \mathbf{u}+\nabla b \times \mathbf{F}-\mathscr{D} \boldsymbol{\omega}_{a}
$$

has an advective constituent $q \mathbf{u}$ and nonadvective constituents that arise from diabatic processes $\mathscr{D}$ :

$$
\mathscr{D} \equiv \frac{\partial b}{\partial t}+\mathbf{u} \cdot \nabla \mathbf{b}
$$


and from frictional forces F. Calculating the divergence of the PV flux in (2), the PV equation becomes

$$
\frac{\partial q}{\partial t}+\mathbf{u} \cdot \nabla q=\nabla \times \mathbf{F} \cdot \nabla b+\omega_{a} \cdot \nabla \mathscr{D}
$$

showing how frictional torques on isopycnal surfaces or gradients of diapycnal mixing in the direction of absolute vorticity change the PV (Marshall and Nurser, 1992).

\subsection{Atmospheric forcing conditions favorable for PV destruction and ITE formation}

To determine what atmospheric forcing conditions are favorable for PV destruction and whether or not friction can play a significant role the formation of low-PV ITEs at ocean fronts, consider integrating (2) over a control volume that encircles a frontal zone in the Northern Hemisphere (i.e. in the discussion that follows, $f$ is taken to be positive, although the results can be easily generalized to the Southern Hemisphere). Application of Gauss' theorem to the integral reveals that reduction of the magnitude of the volume-integrated PV occurs when the net flux of PV through the surface of the control volume is outward. Consider the control volume $\mathscr{V}$ shown in Fig. 1, with an upper surface that coincides with the outcrop window of a front, side surfaces that are isopycnals, and a bottom surface that crosses isopycnals at a depth where it is assumed that the flow, frictional forces, diabatic processes and hence PV fluxes are weak. For such a volume, only the PV flux out of the sea-surface contributes to the change of volume-integrated PV since no PV is fluxed through the isopycnal surfaces of the control volume, in accordance with the "impermeability theorem" of Haynes and McIntyre (1987). Therefore, the PV in the 
control volume will be reduced if the PV flux at the sea-surface is upward.

At the sea-surface, the vertical component of the PV flux is dominated by its nonadvective constituents. For an inertially stable flow, where the vertical component of the absolute vorticity

$$
\zeta_{a b s}=f+\zeta
$$

$(\zeta=\hat{k} \cdot \nabla \times \mathbf{u})$ is positive, diabatic processes will result in an upward PV flux

$$
J_{z}^{\mathscr{D}}=-\zeta_{a b s} \mathscr{D}
$$

when $\mathscr{D}<0$ and diabatic processes reduce the buoyancy. Assuming that $\mathscr{D}$ is dominated by vertical mixing processes so that it can be represented by the convergence of a vertical buoyancy flux, i.e. $\mathscr{D}=-\partial \mathscr{B} / \partial z$, diabatic processes will reduce the PV when when there is a net loss of buoyancy from the ocean to the atmosphere, that is when $\mathscr{B}_{\text {atm }}>0$, where $\mathscr{B}_{\text {atm }}$ is the air-sea buoyancy flux. This result that PV destruction occurs when $\mathscr{B}_{a t m}>0$ is not surprising, since buoyancy loss to the atmosphere triggers convective mixing which reduces the stratification (e.g. Marshall and Schott, 1999).

The vertical component of the nonadvective PV flux associated with frictional forces

$$
J_{z}^{F}=\nabla_{h} b \times \mathbf{F}
$$

is nonzero only if there is a horizontal buoyancy gradient $\nabla_{h} b$, implicating ocean fronts as key regions for frictional PV change. Associating the horizontal buoyancy gradient with a vertically sheared geostrophic flow $\mathbf{u}_{g}$ via the thermal wind relation

$$
\nabla_{h} b=f \frac{\partial \mathbf{u}_{g}}{\partial z} \times \hat{k}
$$


reveals that the nonadvective PV flux associated with frictional forces

$$
J_{z}^{F}=f \frac{\partial \mathbf{u}_{g}}{\partial z} \cdot \mathbf{F}
$$

is upward when the frictional force is in the direction of the geostrophic shear. For wind-forced flows, the frictional force is dominantly in the direction of the wind-stress. Currents at upper-ocean fronts are usually surface intensified so that the surface current of the front is oriented with the geostrophic shear. Therefore, when the wind blows in the direction of the frontal jet, i.e. is in a "down-front" orientation, the conditions are favorable for PV destruction by frictional forces (i.e. $J_{z}^{F}>0$ ).

\subsection{Physical mechanism responsible for PV destruction by winds: two-dimensional numerical experiments}

Down-front winds drive Ekman flow that advects water from the dense to lessdense side of the front, destabilizing the water column. The ensuing convection is characterized by a buoyancy flux whose strength scales with the following quantity

$$
\mathscr{B}_{\text {wind }}=\mathbf{M}_{e} \cdot \nabla_{h} b
$$

(Thomas and Lee, 2005). Mixing by this Ekman-driven convection reduces the stratification and the PV near the sea surface.

The effects of PV destruction by winds are however not limited to the Ekman layer where frictional forces are strongest, but, as a consequence of ageostrophic secondary circulations (ASCs), are felt within a deepening surface layer of nearly zero PV (Thomas, 2005). The two-dimensional frontal zone numerical experiments of Thomas (2005) illustrate the way in which the 
ASCs redistribute PV in the vertical. ASCs subduct low PV from the surface and upwell high PV from the pycnocline which is subsequently destroyed by friction in the Ekman layer. In this way, the ASCs act as a PV pump that extracts PV from the pycnocline, reducing the PV there, and thus deepening the zero-PV surface layer.

The zero-PV surface layer in the experiments of Thomas (2005) was characterized by non-zero stratification and non-zero absolute vorticity (6). To demonstrate how a fluid can have zero PV and non-zero stratification/absolute vorticity, the PV (1) can be split into two parts

$$
q=q_{v e r t}+q_{b c}
$$

one associated with the vertical component of the absolute vorticity and the stratification

$$
q_{v e r t}=\zeta_{a b s} N^{2}
$$

the other attributable to the horizontal components of vorticity and buoyancy gradient

$$
q_{b c}=\frac{\partial u}{\partial z} \frac{\partial b}{\partial y}-\frac{\partial v}{\partial z} \frac{\partial b}{\partial x}
$$

(note that only hydrostatic motions are being considered, so that in (14) terms in the horizontal vorticity involving lateral derivatives of the vertical velocity can be neglected). For a geostrophic flow, it can be shown using the thermal wind relation (9) that (14) reduces to

$$
q_{b c}^{g}=-f\left|\frac{\partial \mathbf{u}_{g}}{\partial z}\right|^{2}=-\frac{1}{f}\left|\nabla_{h} b\right|^{2},
$$

which is a negative definite quantity, indicating that the baroclinicity of the fluid always reduces the PV. If $q \leq 0$ and the flow is nearly geostrophic, then

$$
\left(1+\frac{\zeta}{f}\right) \frac{N^{2}}{\left|\partial \mathbf{u}_{g} / \partial z\right|^{2}} \leq 1 .
$$


For a low Rossby number flow (i.e. $|\zeta| / f \ll 1$ ) then the $q \leq 0$ condition is equivalent to stating that the Richardson number of the geostrophic flow is less than or equal to one. Therefore for strongly baroclinic flows with high vertical shear, the PV can be zero even if $\zeta_{a b s} \neq 0$ or $N^{2} \neq 0$. For such flows, the low PV that results will be referred to as "baroclinically-low" PV.

Low-PV anomalies that characterize ITEs have been associated with anticyclonic vorticity $(\zeta<0)$ and/or weak stratification, not with baroclinically-low PV (e.g. McWilliams, 1985; D'Asaro, 1988). The frontal zone experiments of Thomas (2005) show how down-front winds form low PV, but the low PV that is formed is baroclinically low and hence is distinct from the low PV of ITEs. Therefore, if PV destruction by winds at ocean fronts is to result in the formation of ITEs, then some other mechanism not captured in the simple two-dimensional experiments must come into play. The numerical experiment that will be presented in the next section reveals that down-front winds can indeed drive ITE generation, provided that fully three-dimensional frontal circulations are allowed.

\section{$3 \quad$ Numerical experiment}

\subsection{Configuration}

For the numerical experiment presented in this paper, an initial zonally symmetric density field was chosen that is periodic in the north and south direction over the interval $[0, L]$, is characterized by isolated fronts of width $L_{f}$, and has a pycnocline. An analytical form for the initial condition of the density that 
incorporates these features is

$$
\rho(y, z)=\mathrm{Z}(z)\left(\Delta \rho_{y} \mathrm{Y}(y)-\Delta \rho_{z}\right)+\bar{\rho}(z)+\rho_{c}
$$

where

$$
\begin{gathered}
Y(y)=\left\{\begin{array}{c}
0.5\left[1-\tanh \left(\frac{y}{L_{f}}\right)+\tanh \left(\frac{y-L / 2}{L_{f}}\right)\right] \quad 0 \leq y \leq L / 2 \\
0.5\left[\tanh \left(\frac{y-L / 2}{L_{f}}\right)-\tanh \left(\frac{y-L}{L_{f}}\right)-1\right] L / 2 \leq y \leq L
\end{array}\right. \\
Z(z)=0.5\left[1+\tanh \left(\frac{z+H}{\Delta h}\right)\right], \\
\bar{\rho}(z)=\left\{\begin{array}{c}
0 \geq z_{o}, \\
-\left(\rho_{o} / g\right) \bar{N}^{2}\left(z-z_{o}\right) z \leq z_{o}
\end{array}\right.
\end{gathered}
$$

and $\rho_{c}$ is a constant chosen to be $29.5 \mathrm{~kg} \mathrm{~m}^{-3}$. This constant is purely arbitrary and not taken from observations. It was added for ease in referencing various isopycnal surfaces mentioned later in the text. The specific values of the coefficients used in (16)-(19) are $L_{f}=4 \mathrm{~km}, H=100 \mathrm{~m}, \Delta h=20 \mathrm{~m}$, $\bar{N}^{2}=4.9 \times 10^{-5} \mathrm{~s}^{-2}, z_{o}=-129 \mathrm{~m}, \Delta \rho_{z}=1 \mathrm{~kg} \mathrm{~m}^{-3}$, and $\Delta \rho_{y}=0.5 \mathrm{~kg}$ $\mathrm{m}^{-3}$, which yield a frontal gradient and structure that is modeled after the subpolar front of the Japan/East Sea during the winter (Thomas and Lee, 2005). The maximum surface lateral buoyancy gradient calculated from (16), $|\partial b / \partial y|_{\max }=6.0 \times 10^{-7} \mathrm{~s}^{-2}$, is on the high end of typical values for the lateral buoyancy gradient of upper-ocean fronts. The fluid is on an $f$-plane with the Coriolis parameter set to $f=1 \times 10^{-4} \mathrm{~s}^{-1}$. The density field is accompanied by a geostrophically balanced zonal flow calculated using a level of no motion at the bottom of the model domain. A north-south cross section of the initial conditions is shown in figure 2 .

The numerical experiment was performed using the Regional Oceanic Mod- 
eling System (ROMS) (Shchepetkin and McWilliams, 2005). The simulation was fully three dimensional and was run in a domain of width $L=200 \mathrm{~km}$ in the meridional, $L_{x}=45 \mathrm{~km}$ in the zonal, and depth $500 \mathrm{~m}$ in the vertical. Lateral boundary conditions were periodic. Stress boundary conditions were applied at the upper and lower boundaries for the zonal and meridional velocities. All surface stresses were set to zero except for the zonal stress at $z=0$ which was equal to the wind-stress $\tau_{w}^{x}$. Zero flux boundary conditions were applied to the density at the upper and lower boundaries. So as to adequately resolve frontal features, horizontal grid spacings of $\Delta x=1000 \mathrm{~m}$ and $\Delta y=500 \mathrm{~m}$ were used. The vertical resolution of the model was nonuniform, ranging from $\Delta z=6 \mathrm{~m}$ in the proximity of the surface, to $\Delta z=56 \mathrm{~m}$ near the bottom. A total of 25 vertical levels were utilized. Vertical mixing of momentum and density was accomplished using a version of the K-profile parameterization (Large et al., 1994) scheme that was modified to parameterize Ekman-driven convection (e.g. Thomas, 2005). For numerical stability, biharmonic horizontal diffusion of buoyancy and momentum is included with a hyperviscosity/diffusivity coefficient of $\kappa_{h}=1.7 \times 10^{5} \mathrm{~m}^{4} \mathrm{~s}^{-1}$.

The model is forced by a spatially uniform zonal wind that is turned on impulsively at $t=0$, kept at a constant value of $\tau_{w}^{x}=0.2 \mathrm{~N} \mathrm{~m}^{-2}$, then turned off at $t=5$ inertial periods $(\sim 3.6$ days). The magnitude and temporal variation of the wind-stress is meant to be an idealized representation of the passage of a moderately strong wind-event. The experiment is integrated until $t=10$ inertial periods so that both the spin-up and relaxation of the front during and after the wind forcing can be investigated. For the orientation of the wind $\left(\tau_{w}^{x}>0\right)$, the fronts centered initially at $y=L / 2$ and $y=0, L$ are forced by down-front and up-front winds, respectively. By studying the response of 
the fluid to the winds at the separate fronts, the numerical experiment can be used to examine both the destruction and formation of PV by wind-driven frictional forces, although the primary emphasis of the analysis in this paper will be on PV destruction.

\subsection{Model results}

When the wind-stress is on, southward Ekman transport advects the surface density field. For the first few inertial periods, the flow is two-dimensional, but after $\sim 2.5$ inertial periods, the front that was initial centered at $y=100$ $\mathrm{km}$ becomes unstable to three-dimensional instabilities (figure 3). Calculations of the energetics of the flow (not shown) reveal that the dominant source of energy for the instabilities is the release of available potential energy from the mean flow. The meandering motions can hence be classified as a form of baroclinic instability, similar to the submesoscale mixed layer instabilities described in Boccaletti et al. (2007) and Capet et al. (2008). In contrast to the front with the eastward frontal jet, the front that was initially at $y=0,200$ $\mathrm{km}$, remains two-dimensional throughout the experiment. While the wind is blowing, this front translates to the south and, at its leading edge, a stratified layer forms in the upper $30 \mathrm{~m}$. This restratification is a consequence of Ekman advection of lighter water over dense.

At the front in the center of the domain, the downfront wind forcing leads to the formation of low-PV water, as can be seen in the maps of the PV on an isopycnal surface that outcrops at the front (figure 4). As the front forms meanders, the frontal vertical circulation upwells water with high PV from the pycnocline and subducts low-PV surface water. As is typical of meandering 
fronts, subduction occurs on the down-stream side of meander crests where the flow tends to be frontogenetic and the ageostrophic circulation is strongest. The spatial separation of the two downwelling centers results in the formation of two distinct low-PV water masses. The first water mass (located near $x=20$ $\mathrm{km}$ at $t=4.8$ inertial periods) is stretched and sheared by the deformation field of the flow and by $t=10$ inertial periods forms an elongated ribbon of low PV. The second low-PV water mass survives the shearing motion of the flow, wrapping into a coherent circular vortex with properties characteristic of an ITE.

\subsubsection{General description of the ITE}

A meridional cross-section of the density field and zonal velocity striking through the core of the low-PV vortex is shown in figure 5. The vortex has the classic structure of an ITE, with convex isopycnals, anomalously weak stratification relative to its surroundings, a velocity field with an interior maximum, and anticyclonic circulation. The ITE is a submesoscale feature, with a radius $r_{l} \approx 6 \mathrm{~km}$ and half-width (i.e. the maximum elevation of the lens above its center) $H_{l} \approx 32 \mathrm{~m}$. A nondimensional measure of the ITEs aspect ratio is the Burger number

$$
B u=\frac{N_{b} H_{l}}{f r_{l}}
$$

where $N_{b}=9.8 \times 10^{-3} \mathrm{~s}^{-1}$ is the buoyancy frequency of the surrounding background fluid. Given the dimensions of the ITE, the Burger number of the vortex is $B u \approx 0.52$. An analytical model for anticyclonic lenses predicts (and laboratory experiments confirm) that there is a linear relationship between a lens' Burger number and Rossby number $R o=|\zeta| /(2 f)$, such that for lenses with $B u \approx 0.5, R o \approx 0.5$ (Gill, 1981; Hedstrom and Armi, 1988). 
This result implies that in the center of the vortex the absolute vorticity (6) should be approximately zero. In an ITE with such a large Rossby number, the analytical model for SCVs of McWilliams (1985) predicts that the centrifugal force is critical to the leading order radial force balance. To see if these analytical predictions accurately describe the ITE of the numerical experiment, radial profiles of the azimuthal velocity, vertical relative vorticity, and terms in the radial force balance within the ITE were calculated and are presented in figure 6. Radial profiles of variables were calculated by determining the center of the vortex $\left(x_{o}, y_{o}\right)$, calculating the radial coordinate $r=\sqrt{\left(x-x_{o}\right)^{2}+\left(y-y_{o}\right)^{2}}$, creating radial bins separated by $1 \mathrm{~km}$, then binning the variables. The vortex center was taken to be the location where the speed of the horizontal flow approaches zero and the azimuthal angle was calculated as $\theta=\tan ^{-1}\left[\left(y-y_{o}\right) /\left(x-x_{o}\right)\right]$.

The azimuthal velocity, $u_{\theta}=v \cos \theta-u \sin \theta$, is negative and its magnitude peaks near $r \sim 6 \mathrm{~km}$ at a value of $\left|u_{\theta}\right| \approx 0.25 \mathrm{~m} \mathrm{~s}^{-1}$, figure 6(a). The approximate linear increase in speed for $r<6 \mathrm{~km}$ indicates that the fluid is in a solid body rotation in the interior of the vortex. The clockwise rotation of the fluid in the vortex core corresponds to a relative vertical vorticity, figure $6(b)$, that approaches $\zeta=-f$, implying that the Rossby number of the ITE $R o=|\zeta| /(2 f) \approx 0.5$, consistent with the prediction of Gill (1981).

The leading order radial force balance for SCVs of McWilliams (1985) is given by the cyclogeostrophic relation:

$$
\frac{u_{\theta}^{2}}{r}=\frac{1}{\rho_{o}} \frac{\partial p}{\partial r}-f u_{\theta}
$$

where the term on the left hand side is the centrifugal force and $p$ is the pressure. Radial profiles of the left and right hand sides of (21) show a general 
correspondence, figure $6(c)$, indicating that the leading order force balance in the ITE is well described by the cyclogeostrophic relation (21), in accordance with the model of McWilliams (1985).

\subsubsection{Potential vorticity budget on the ITE isopycnal surface}

To determine quantitatively the relative contributions of friction and diabatic processes in the generation of the low-PV water critical to the formation of the ITE, a potential vorticity budget was constructed on the isopycnal surface of the ITE. The budget was formed by integrating the various terms in (5):

$$
\begin{aligned}
\frac{\partial}{\partial t} \iiint_{\mathscr{V}} q \mathrm{~d} V= & -\underbrace{\iiint_{\mathscr{V}} \mathbf{u} \cdot \nabla q \mathrm{~d} V}_{\text {ADV }}+\underbrace{\iiint_{\mathscr{V}} \nabla \times \mathbf{F} \cdot \nabla b \mathrm{~d} V}_{\text {FRIC }} \\
& +\underbrace{\iiint_{\mathscr{V}} \omega_{a} \cdot \nabla \mathscr{D} \mathrm{d} V}_{\text {DIA }}+\underbrace{\iint_{\rho_{u}} q u^{\rho \perp} \mathrm{d} S-\iint_{\rho_{l}} q u^{\rho \perp} \mathrm{d} S}_{\text {MOTION }}
\end{aligned}
$$

over a control volume $\mathscr{V}$ bounded on its sides by the $\rho_{u}=28.5$ and $\rho_{l}=$ $28.3 \mathrm{~kg} \mathrm{~m}^{-3}$ isopycnal surfaces, on the top (at $z=-6 \mathrm{~m}$ ) by the outcrop area between $\rho_{u}$ and $\rho_{l}$, and on the bottom by the surface formed by the intersection of the $\rho_{u}$ and $\rho_{l}$ isopycnals with the vertical plane at $y=50 \mathrm{~km}$. The first three terms on the right hand side (RHS) of (22) are the advective (ADV), frictional (FRIC), and diabatic (DIA) contributions to the change in the volume integrated PV. The last term on RHS (MOTION) arises from the motion of the side isopycnal surfaces of the control volume that move at a velocity $u^{\rho \perp}$. Time integrals of the terms on the RHS of (22) are shown in figure 7 along with the timeseries of the volume integrated PV on the ITE isopycnal layer. While the wind is blowing, the value of the volume integrated PV reduces, decreasing by nearly $80 \mathrm{~m}^{3} \mathrm{~s}^{-3}$ in $t=5$ inertial periods. At $t=5$ inertial periods, the time integral of the frictional and diabatic terms are 
both negative, with the frictional term contributing to nearly $90 \%$ of the net decrease in the volume integrated PV. This result indicates that it is frictional not diabatic PV reduction that generates the low-PV water and ITE in the numerical experiment.

Before the wind is shut-off, i.e. $t<5$ inertial periods, the advection and control volume motion terms tend to cancel each other and hence do not play a significant role in the PV budget. After $t>5$ inertial periods, however, the advective term dominates the PV budget and leads to an increase in the volume integrated PV. For an advective PV increase to occur, there must be a net inward advective flux of PV through the surfaces of the control volume. Closer inspection of the structure of the advective PV flux reveals that high PV from the region between the upper surface of the control volume and the sea surface is advected into $\mathscr{V}$. This high $\mathrm{PV}$ is generated by the frictional spin-down of the frontal jet after the wind is turned off. The frictional force responsible for this deceleration is strongest above $z=-6 \mathrm{~m}$ and therefore does not directly affect the PV budget in the control volume such that the time integral of FRIC stays relatively unchanged after $t=5$ inertial periods, figure 7 .

The extraction of PV from the control volume by friction for $t<5$ inertial periods occurs at the top surface of $\mathscr{V}$ via an upward frictional PV flux. The strength of the frictional PV flux is not spatially uniform but varies across the front. To show this, the net frictional PV flux through the outcrop area at $z=-3 \mathrm{~m}$ :

$$
\mathscr{J}_{z}^{F}=\left.\int_{x=0}^{x=L_{x}} \int_{y=y_{l}}^{y=y_{u}} J_{z}^{F}\right|_{z=-3 m} \mathrm{~d} y \mathrm{~d} x
$$

$\left(y_{u}, y_{l}\right.$ are the meridional locations of the isopycnal surfaces that bound the 
outcrop) was calculated for isopycnal layers separated by $0.025 \mathrm{~kg} \mathrm{~m}^{-3}$ and with densities from 28.225 to $28.7 \mathrm{~kg} \mathrm{~m}^{-3}$, and is plotted in figure 8 along with minus its time integral, i.e. $-\int_{s=0}^{s=t} \mathscr{J}_{z}^{F}$ ds. During the times when the low-PV water masses are being formed between $t=3.5-5$ inertial periods, $\mathscr{J}_{z}^{F}$ has a maximum between the density range of $28.3-28.5 \mathrm{~kg} \mathrm{~m}^{-3}$. Correspondingly,

the time integral of minus $\mathscr{J}_{z}^{F}$ has a minimum in these density classes. As can be seen in figure 5, the bulk of the ITE's structure is found between the 28.3 and $28.5 \mathrm{~kg} \mathrm{~m}^{-3}$ isopycnal surfaces. This suggests that the isopycnal distribution of the surface frictional PV flux along with the initial conditions of the surface PV field contribute to determining on which isopycnal layer the ITE forms.

Notice that (23) was calculated at $z=-3 \mathrm{~m}$, at a vertical level above the upper boundary of the control volume used in the PV budget (22). This level was within the frictional boundary layer both before and after the wind was turned off and the front restratified. At this depth, the frictional PV flux reverses sign after $t=5$ inertial periods while the frontal jet was spinning down. As alluded to above, this process generated the high PV that was advected into the isopycnal layer of the control volume $\mathscr{V}$.

\subsubsection{Lagrangian analysis of vortex formation}

Although it has been determined that friction is the process that generates the low-PV water which is the materia prima of the ITE, the mechanism by which this water forms into a coherent anticyclonic vortex has yet to be elucidated. All that has been shown thus far is that friction generates a mass of $q \approx 0$ water. From the decomposition of the PV (12), it can be seen that depending 
on the fluid's stratification, vertical vorticity, and baroclinicity, there are an infinite number of ways in which a fluid can be in a state of zero PV. The various configurations that a $q=0$ fluid can be in can be broadly categorized into three cases

(i) no stratification $\left(N^{2}=0, \zeta_{a b s} \neq 0,\left|\nabla_{h} b\right|^{2}=0\right)$

(ii) vortically-low PV $\left(N^{2} \neq 0, \zeta_{a b s}=0,\left|\nabla_{h} b\right|^{2}=0\right)$

(iii) baroclinically-low $\operatorname{PV}\left(N^{2} \neq 0, \zeta_{a b s} \neq 0,\left|\nabla_{h} b\right|^{2}=N^{2} f \zeta_{a b s}\right)$

The fully developed ITE of the numerical experiment is most closely characterized by case (ii). To determine how this particular $q \approx 0$ configuration was selected in the experiment, a Lagrangian analysis of the PV, vertical vorticity, and baroclinicity of fluid parcels embedded in the ITE was performed.

A total of 35 Lagrangian particles were released at $t=4.8$ inertial periods in the particular low-PV plume that coalesced into the ITE, see figure 4. The position of each particle was calculated online during the model run. Lagrangian timeseries of the various quantities of interest described below were calculated offline by interpolating each quantity to the locations of the particles. So as to emphasize the robust properties of the solution, the timeseries were then averaged over all fluid particles. This ensemble average is denoted by \langle\rangle .

To establish which zero PV configuration fluid parcels in the ITE were in, Lagrangian timeseries of $\langle q\rangle$ and its two parts (12) $\left\langle q_{v e r t}\right\rangle$ and $\left\langle q_{b c}\right\rangle$ were constructed, figure 9 top panel. At the start of the timeseries, the ensemble averaged PV $\langle q\rangle$ is negative. This low PV is a consequence of the baroclinicity of the fluid, as $\left\langle q_{b c}\right\rangle<0$ is larger in magnitude than the positive $\left\langle q_{v e r t}\right\rangle$. Thus, at this time, the low-PV configuration most resembles case (iii), although the strong anticyclonic vorticity $(\langle\zeta\rangle \approx-0.8 f)$ that the particles have at $t=4.8$ 
inertial periods preconditions the fluid to having baroclinically-low PV by reducing the absolute vorticity and $\left\langle q_{\text {vert }}\right\rangle$. This strong anticyclonic vorticity is a consequence of the initial conditions for the frontal jet, combined with the fact that the particles and low-PV water mass were located on the anticyclonic side of the jet before they were subducted.

From $t=5-6$ inertial periods, as the particles are subducted into the interior of the fluid, both $\left\langle q_{v e r t}\right\rangle$ and $\left\langle q_{b c}\right\rangle$ decay to zero. Decomposing $\left\langle q_{\text {vert }}\right\rangle$ into parts associated with the planetary $\left\langle f N^{2}\right\rangle$ and relative $\left\langle\zeta N^{2}\right\rangle$ vorticity, reveals that the $\left\langle q_{\text {vert }}\right\rangle \approx 0$ state is attained because the relative vorticity decreases, not because the fluid becomes unstratified. During this time, the ensembleaveraged vertical vorticity $\langle\zeta\rangle \rightarrow-f$, driving the absolute vorticity and $\left\langle q_{\text {vert }}\right\rangle$ to zero, figure 9 middle panel. Therefore, as the low-PV water is subducted, the fluid shifts from configurations (iii) to (ii).

The shift in the $q=0$ configurations results from changes in the vertical vorticity and lateral (primarily north-south) buoyancy gradient of the fluid. These changes are governed by the following equations

$$
\begin{gathered}
\frac{\mathrm{D} \zeta}{\mathrm{D} t}=(f+\zeta) \frac{\partial w}{\partial z}+\frac{\partial u}{\partial z} \frac{\partial w}{\partial y}-\frac{\partial v}{\partial z} \frac{\partial w}{\partial x} \\
\frac{\mathrm{D}}{\mathrm{D} t}\left(\frac{\partial b}{\partial y}\right)=-\frac{\partial b}{\partial z} \frac{\partial w}{\partial y}-\frac{\partial b}{\partial x} \frac{\partial u}{\partial y}-\frac{\partial b}{\partial y} \frac{\partial v}{\partial y}
\end{gathered}
$$

Frictional and diabatic terms do not appear in (24) and (25) as they are assumed to be negligible during the subduction of the low-PV fluid to the interior of the fluid. The various terms on the right hand sides of (24) and (25) were evaluated on each Lagrangian particle, ensemble-averaged, then integrated in time to yield the following quantities: 


$$
\begin{aligned}
\mathrm{STR} & =\int_{t_{o}}^{t}\left\langle(f+\zeta) \frac{\partial w}{\partial z}\right\rangle d t^{\prime}+\zeta_{o} \\
\mathrm{TILT} & =\int_{t_{o}}^{t}\left\langle\frac{\partial u}{\partial z} \frac{\partial w}{\partial y}-\frac{\partial v}{\partial z} \frac{\partial w}{\partial x}\right\rangle d t^{\prime}+\zeta_{o} \\
\mathrm{TOT}_{\zeta} & =\mathrm{STR}^{\prime} \mathrm{TILT}-\zeta_{o} \\
\mathrm{DVADV} & =-\int_{t_{o}}^{t}\left\langle\frac{\partial b}{\partial z} \frac{\partial w}{\partial y}\right\rangle d t^{\prime}+\left.\left\langle\frac{\partial b}{\partial y}\right\rangle\right|_{t=t_{o}} \\
\mathrm{SHEAR}^{t} & =-\int_{t_{o}}^{t}\left\langle\frac{\partial b}{\partial x} \frac{\partial u}{\partial y}\right\rangle d t^{\prime}+\left.\left\langle\frac{\partial b}{\partial y}\right\rangle\right|_{t=t_{o}} \\
\mathrm{SQZ}^{t} & =-\int_{t_{o}}^{t}\left\langle\frac{\partial b}{\partial y} \frac{\partial v}{\partial y}\right\rangle d t^{\prime}+\left.\left\langle\frac{\partial b}{\partial y}\right\rangle\right|_{t=t_{o}} \\
\mathrm{TOT}_{\partial b / \partial y} & =\mathrm{DVADV}+\mathrm{SHEAR}+\mathrm{SQZ}-\left.2\left\langle\frac{\partial b}{\partial y}\right\rangle\right|_{t=t_{o}}
\end{aligned}
$$

where $t_{o}$ is the time at which the particles were released and $\zeta_{o}=\left\langle\zeta\left(t_{o}\right)\right\rangle$. These quantities are shown in figure 9 along with the timeseries of $\langle\zeta\rangle$ and $\langle\partial b / \partial y\rangle$ so as to determine which of processes are responsible for the reduction of baroclinicity and absolute vorticity.

The generation of anticyclonic vorticity in the core of the ITE is due to the TILT term, which represents the tilting of the horizontal vorticity vector downward by differential vertical motions. For vortex tilting to be effective at modifying $\zeta$, there must exist both a vertically sheared horizontal flow and laterally varying upwelling and downwelling. The front, with its strong baroclinicity and meander-driven vertical circulation, supplies both of these features. The tendency of the TILT term to create negative vorticity is partially compensated by vortex stretching, STR. The positive value of STR indicates that as the low-PV water is subducted, vortex columns are stretched, such that vortex stretching hinders rather than augments the generation of the ITE's anticyclonic vorticity.

As the low-PV water of the ITE coalesces, the meridional buoyancy gradient $\langle\partial b / \partial y\rangle$ increases in value but decreases in magnitude, figure 9 bottom 
panel. The increase in $\langle\partial b / \partial y\rangle$ is due to both the DVADV and SHEAR terms, which represent differential vertical advection of buoyancy and the conversion of east-west buoyancy gradients to north-south buoyancy gradients by a meridionally-sheared zonal flow, respectively. During the phase when the baroclinic contribution to the PV rapidly diminishes, i.e. between 5-6 inertial periods, the increase in $\langle\partial b / \partial y\rangle$ is dominated by DVADV, indicating that the primary cause for the reduction in baroclinicity is the slumping of isopycnals by vertical motions. DVADV is somewhat counteracted by the squeezing together of isopycnals by convergent flow, i.e. term SQZ, but not completely. For $t>7$ inertial periods, the reduction in DVADV is partially compensated by the increase in SHEAR, such that the net tendency of the north-south buoyancy gradient $\mathrm{TOT}_{\partial b / \partial y}$ slowly decreases with time.

The Lagrangian analysis above has demonstrated the importance of differential vertical motions, through the tilting of isopycnals and the horizontal vorticity vector, in the reduction of baroclinicity and development of anticyclonic vorticity within the ITE. The differential vertical motions in the simulation are associated with frontal meanders. It can be therefore concluded that meanders play a critical role in the formation of the ITE as they provide both a mechanism for the subduction of the low-PV fluid into the interior and for the conversion of the $q \approx 0$ water from being baroclinically low to being vortically low with zero absolute vorticity.

\subsubsection{Effect of the ITE on the zonal mean flow}

The subduction of low-PV from the surface and upwelling of high PV from the pycnocline during frontal meandering and ITE formation that is evident in fig- 
ure 4 suggests that there is a correlation between PV anomalies and the velocity field. Such a correlation would be associated with an eddy PV flux. Eddy PV fluxes on isopycnal surfaces are important because they are equivalent to forces and thus can accelerate large-scale flow and/or drive eddy-induced transport ("bolus") velocities that modify large-scale tracer-fields via advection (Andrews et al., 1987; Greatbatch, 1998). To investigate the impact that the ITE has on the large-scale, zonally-averaged flow, a zonally-averaged zonal momentum budget in isopycnal coordinates will be formulated and applied to the numerical experiment.

The zonal momentum equation in isopycnal coordinates is

$$
\frac{\partial u_{\rho}}{\partial t}-q_{\rho} z_{\rho} v_{\rho}=-\frac{1}{\rho_{o}} \frac{\partial B}{\partial x}+X_{\rho}
$$

where $z_{\rho}$ is the thickness of the isopycnal layer, $B$ is the Bernoulli function, $X_{\rho}$ is the layer zonal frictional force, $\left(u_{\rho}, v_{\rho}\right)$ are the zonal and meridional components of the velocity on the layer, and

$$
q_{\rho}=\frac{f+\zeta_{\rho}}{z_{\rho}}
$$

is the layer PV (Greatbatch, 1998). Averaging (33) in the zonal direction results in the following equation

$$
\frac{\partial \overline{u_{\rho}}}{\partial t}-\hat{q}_{\rho} \overline{z_{\rho}} \hat{v}_{\rho}=\overline{z_{\rho} q_{\rho}^{\prime \prime} v_{\rho}^{\prime \prime}}+\overline{X_{\rho}}
$$

where the over bar denotes a zonal average $\overline{()}=\left(1 / L_{x}\right) \int_{0}^{L_{x}} \mathrm{~d} x$, hats the thickness-weighted isopycnal average, e.g.

$$
\hat{v}_{\rho}=\frac{\overline{v_{\rho} z_{\rho}}}{\overline{z_{\rho}}},
$$

and double primes the deviation from the thickness-weighted isopycnal average, e.g. $v_{\rho}^{\prime \prime}=v_{\rho}-\hat{v}_{\rho}$ (Greatbatch, 1998). 
To calculate the various terms in (35) using the output from the $z$-coordinate ROMS model, isopycnally-averaged quantities were calculated by doing the following. At each horizontal position the vertical locations of the upper $\left(z_{u}\right)$ and lower $\left(z_{l}\right)$ isopycnal surfaces that bound the isopycnal layer are found and the layer thickness $z_{\rho}=z_{u}-z_{l}$ is computed. For each variable $A$, a layer average is calculated as

$$
A_{\rho}=\frac{1}{z_{\rho}} \int_{z_{l}}^{z_{u}} A \mathrm{~d} z
$$

from which both the zonal and thickness-weighted, isopycnal-average can be formed.

A momentum budget for $\overline{u_{\rho}}$ on the layer bounded by the 28.3 and $28.5 \mathrm{~kg}$ $\mathrm{m}^{-3}$ isopycnal surfaces (with a difference in buoyancy of $\Delta b=0.0019 \mathrm{~m} \mathrm{~s}^{-2}$ ) was computed and is shown in figure 10. Early in the experiment, before the front goes unstable to three-dimensional instabilities, the primary balance is between acceleration, the effective Coriolis force, $\hat{q}_{\rho} \overline{z_{\rho}} \hat{v}_{\rho}$ (note that minus the effective Coriolis force is plotted in the figure), and friction, i.e. a timedependent Ekman balance is present. The importance of time-dependence is evident in the oscillation of the acceleration and Coriolis terms at close to the inertial frequency and is a consequence of the impulsive start to the windforcing. After the wind is shut off ( $t>5$ inertial periods), the frictional force is negligible and does not play a role in the interior momentum balance. Before this time, at $t \sim 3.5$ inertial periods, the front goes unstable, and the eddy PV flux, $\overline{z_{\rho} q_{\rho}^{\prime \prime} v_{\rho}^{\prime \prime}}$, amplifies to become the dominant term in the momentum equation. The eddy PV flux has a similar meridional and temporal structure to the effective Coriolis force, indicating that the two forces tend to balance. This balance is not perfect, however, and results in net acceleration of the zonal mean flow. 
The maximum in the eddy PV flux propagating to the south, see figure 10(c), is associated with the ITE. The manner in which the circulation of the ITE drives a PV flux is illustrated in figure 11(c). Comparing the structures of the anomalies in the PV, $q_{\rho}^{\prime \prime}$, and velocity, $v_{\rho}^{\prime \prime}$, reveals that at the maximum in the eddy PV flux (which is at $y \approx 75 \mathrm{~km}$ at this time), the zero in $v_{\rho}^{\prime \prime}$ is displaced to the west of the minimum PV anomaly. This yields a correlation between the ITE's southward (northward) flow and the low (high) PV anomaly and drives a net flux of PV. The eddy PV flux is thus a consequence of the asymmetry between the ITE's horizontal circulation and PV distribution. That is, if streamlines of the ITE were perfectly aligned with PV contours, then the ITE would not advect PV and no eddy PV flux would be induced.

The maximum eddy PV flux tends to occur in the proximity of the minimum in the thickness averaged PV where $\partial \hat{q}_{\rho} / \partial y=0$, see figures $11(a)-(b)$ and 12(a)-(b). This suggests that the eddy PV flux does not follow a simple downgradient form:

$$
\overline{z_{\rho} q_{\rho}^{\prime \prime} v_{\rho}^{\prime \prime}}=-\overline{z_{\rho}} \kappa \frac{\partial \hat{q}_{\rho}}{\partial y}
$$

where $\kappa>0$ is an effective eddy diffusivity. Eddy PV fluxes are only expected to be purely downgradient in statistically steady flows in regions where the enstrophy (i.e. the variance of the PV) is dissipated, advective and temporal changes in the enstrophy are negligible, and the PV is not being modified by external forcing (Rhines and Holland, 1979; Wilson and Williams, 2003). The experiment presented here is forced and describes a transient process with an eddy (and hence enstrophy) field that exhibits spatial variations over the width of the front. Therefore, it is not surprising that the eddy PV flux in the simulation does not appear to follow a down-gradient form. 
What appears to set the magnitude and northward direction of the eddy PV flux in the proximity of the ITE is the surface frictional PV flux during the period of wind forcing. At the frontal outcrop, down-front winds drive an upward frictional PV flux at the sea-surface. To the south and in the interior of the fluid where isopycnal surfaces are nearly horizontal, a PV flux parallel to isopycnals and in the same direction as $J_{z}^{F}$ at the surface would be directed to the north, which is the direction of the eddy PV flux. To compare the magnitude of the eddy PV flux to $J_{z}^{F}$, the net eddy advective flux of PV through a surface bounded above and below by the 28.3 and $28.5 \mathrm{~kg} \mathrm{~m}^{-3}$ isopycnals and located at the eddy PV flux maximum, $y=y_{\max }$, was calculated as

$$
\left.\mathscr{J}_{y}^{e}\right|_{y=y_{\max }}=\left.\Delta b \int_{x=0}^{x=L_{x}} \overline{z_{\rho} q_{\rho}^{\prime \prime} v_{\rho}^{\prime \prime}}\right|_{y=y_{\max }} \mathrm{d} x=\left.\Delta b L_{x} \overline{z_{\rho} q_{\rho}^{\prime \prime} v_{\rho}^{\prime \prime}}\right|_{y=y_{\max }} .
$$

and is plotted in figure $12(c)$ along with $\mathscr{J}_{z}^{F}(23)$, where $y_{u}, y_{l}$ in (23) are the locations of the 28.5 and $28.3 \mathrm{~kg} \mathrm{~m}^{-3}$ surfaces at the outcrop. Also plotted in the figure is a scaling for the integrated surface frictional PV flux. The scaling is estimated as follows. Assuming that the frictional force is dominantly in the zonal direction and its magnitude can be approximated as $F_{x} \approx \tau_{w}^{x} / \rho_{o} \delta_{e}$ $\left(\delta_{e}=0.4 \sqrt{\tau_{w}^{x} / \rho_{o}} / f\right.$ is the Ekman layer depth), then (23) can be scaled as

$$
\left[\mathscr{J}_{z}^{F}\right]=\frac{\tau_{w}^{x}}{\rho_{o} \delta_{e}} \int_{x=0}^{x=L_{x}} \int_{y=y_{l}}^{y=y_{u}} \frac{\partial b}{\partial y} \mathrm{~d} y \mathrm{~d} x=\frac{\tau_{w}^{x} \Delta b L_{x}}{\rho_{o} \delta_{e}}
$$

Notice that only the total buoyancy difference $\Delta b$ across the frontal outcrop and not an average measure of the meridional buoyancy gradient comes into play in (39), since $y_{l}$ and $y_{u}$ coincide with the locations of the bounding isopycnals of the outcrop. The timeseries of the integrated PV fluxes reveal that while the wind-stress is on, $\mathscr{J}_{z}^{F}$ fluctuates, but scales with (39), figure $12(c)$. After the front goes unstable, the eddy PV flux $\left.\mathscr{J}_{y}^{e}\right|_{y=y_{\max }}$ grows, peaking at $t \approx 4.5$ inertial periods, then decreases to a quasi-steady value very close to 
the scaling $\left[\mathscr{J}_{z}^{F}\right]$. These results suggest that the eddy PV flux associated with the ITE is set by the wind-stress forcing at the frontal outcrop but is nonlocal in time and space as the eddy PV flux is nonzero both away from the region and after the time-period of forcing.

The partial balance of the eddy PV flux and the effective Coriolis force results in a net southward along-isopycnal flow $\hat{v}_{\rho}<0$ at the meridional location of the ITE, figure $13(a)$. To the south of the PV minimum, $\hat{v}_{\rho}$ decays to zero. This flow convergence results in the thickening of the isopycnal layer. At the same time, the spatial variation in the acceleration of the zonal mean flow leads to the generation of anticyclonic vorticity south of the ITE, figure $10(a)$. The thickening of the layer and the formation of anticyclonic vorticity reduces the thickness averaged PV, $\hat{q}_{\rho}=\left(\overline{\zeta_{\rho}}+f\right) / \overline{z_{\rho}}$, south of the ITE and allows for the southward propagation of the PV minimum, figure 12(a). The thicknessweighted, isopycnally-averaged meridional velocity consists of two parts, an eddy transport or bolus velocity, $v_{\rho}^{*}$, and a zonal mean, $\overline{v_{\rho}}$, i.e.

$$
\hat{v}_{\rho}=\overline{v_{\rho}}+v_{\rho}^{*}
$$

(Greatbatch, 1998). The structure of the zonal mean meridional velocity $\overline{v_{\rho}}$ is dominated by inertial oscillations and, for the period of wind-forcing, Ekman flow, figure 13(b). Comparing figures 13(c)-(d) it can be seen that the bolus velocity follows the eddy PV flux and has a magnitude approximately given by $\left|v_{\rho}^{*}\right| \sim\left|\overline{z_{\rho} q_{\rho}^{\prime \prime} v_{\rho}^{\prime \prime}}\right| / f .{ }^{1}$ This result, combined with the fact that the magnitude

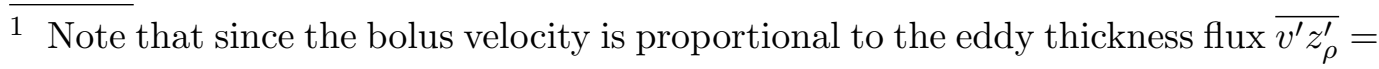
$v_{\rho}^{*} \bar{z}_{\rho}$ (primes denote deviations from the zonal mean), the correspondence between the eddy PV flux and the bolus velocity implies that eddy PV flux is well captured by the thickness flux. This correspondence is to be expected for small amplitude 
of the eddy PV flux is set by the scaling of the surface frictional PV flux (39), can be used to derive a scaling for the bolus velocity

$$
\left[v_{\rho}^{*}\right]=\frac{\left[\mathscr{J}_{z}^{F}\right]}{f \Delta b L_{x}}=\frac{\tau_{w}^{x}}{\rho_{o} f \delta_{e}}
$$

which is identical to the scaling for the Ekman flow. Note that the wind-stress that is used in (41) is the wind-stress that forces the front when the ITE was formed. This again emphasizes the nonlocal nature of the ITE's eddy PV flux and consequently its bolus velocity.

\section{Conclusions}

A numerical experiment capable of resolving submesoscale processes has illuminated a mechanism by which ITEs can form at wind-forced upper ocean fronts. The simulation suggests that ITE formation is facilitated when winds have a component directed down-front, as wind-driven friction reduces the PV within the frontal outcrop, creating low-PV water that is the defining substance of ITEs. Three-dimensional frontal instability and meanders play an important role in the ITE generation mechanism as they drive vertical circulation that subducts the low-PV water into the interior of the ocean. The coalescence of this water into a vortex critically depends on differential vertical motions associated with frontal meanders. Differential vertical motions tilt isopycnal surfaces and horizontal vorticity to reduce both the baroclinicity and absolute vorticity of the fluid. By this process, the absolute vorticity is

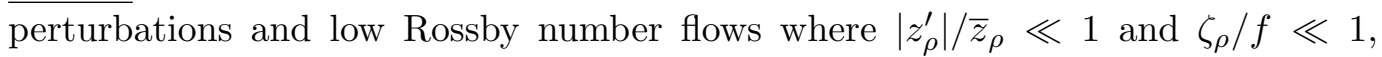
therefore it is somewhat surprising that this result holds for the strongly nonlinear flows simulated in the experiment. 
driven to a value close to zero, at which point a strong anticyclonic circulation surrounds the low-PV water mass, maintaining it as a coherent vortex with properties characteristic of an ITE. The generation of a coherent circular vortex by this mechanism is not guaranteed to occur for all subducted low PV water masses, however, as strain in the large-scale velocity field can disrupt the process and result in the formation of elongated ribbons of low PV.

The ITE generation mechanism described above has both similarities and differences to those proposed in the literature. The mechanism is distinct from that put forth by McWilliams (1985) in that it attributes the creation of the low-PV water to friction rather than diabatic processes. This is not to say that diabatic processes are unlikely to play a role in ITE formation at fronts. If the numerical experiment had additionally been forced by buoyancy loss, a diabatic surface PV flux would have been induced that would have augmented the frictional PV flux, and perhaps supplemented the supply of low-PV water to the ITE. The ITE generation mechanism also differs from McWilliams' in that it ascribes the formation of coherent structures to frontal meandering rather than to inherent spatial variability in the forcing. In this sense, the mechanism is similar to that suggested by Spall (1995). However, whereas Spall (1995) invoked vortex squashing as the governing physics for generating the anticyclonic vorticity of the ITE, the mechanism presented here relies on tilting of the horizontal vorticity of the frontal jet. Nevertheless, vortex squashing and tilting are not mutually exclusive processes, and it is likely that ITE formation in other situations involves some combination of both processes. The mechanism is also similar to that proposed by D'Asaro (1988) in that frictional torques are the key players in the generation of the ITE's low PV. However, the frictional torque of D'Asaro's mechanism is vertical 
and accelerates the anticyclonic vorticity of the ITE directly, while in this mechanism it is the horizontal frictional torque that creates the baroclinically low-PV water that subsequently re-expresses itself as zero absolute vorticity fluid.

The ITE formation mechanism described in this paper requires that the isopycnal surface on which the ITE forms outcrops, and outcrops in a region of strong baroclinicity and wind-forcing. Therefore, SCVs that are found in the abyss, on isopycnals that do not outcrop, could not have been formed by wind-driven PV destruction, but could have have been generated by the mechanisms of McWilliams or D'Asaro, for example. These mechanisms could also be active on isopycnals that outcrop at the sea-surface. However, for vortices with core water with characteristics of surface water (e.g. elevated levels of dissolved oxygen) it is less likely that patchy mixing in the interior or topographic interactions are responsible for their generation. For ITEs that originate at the surface, the low PV water that constitutes their centers could have been created by surface buoyancy fluxes or wind-stress, or more likely, a combination of both (since periods of wind-forcing are often accompanied by buoyancy fluxes). One might ask, under what conditions it is more likely that ITEs form due to the winds versus buoyancy loss? A metric for the relative contributions of diabatic processes and friction to the formation of ITEs is the ratio of the surface frictional to diabatic PV fluxes. As shown in Thomas (2005), $J_{z}^{F} / J_{z}^{\mathscr{D}}$ scales as the the ratio of the wind-driven buoyancy flux, i.e. (11), to the atmospheric buoyancy flux. At strongly baroclinic fronts, and for typical wind-stress magnitudes, this ratio can easily be greater than one, suggesting that for ITEs that form in such regions, wind-driven destruction of PV is likely to play a role in their formation. It might be argued, that the wind-driven mechanism is 
less plausible than the other mechanisms since it is sensitive to the direction of the wind-stress relative to the orientation of the front. However, a numerical experiment described in Thomas (2007), with the same initial conditions as the experiment presented here, yet forced with a wind-stress whose orientation rotates with time, similarly exhibits ITE formation via wind-driven PV destruction and frontal meandering, suggesting that the mechanism does not require the wind's orientation to be fixed in the down-front direction.

The numerical experiment revealed that the ITE, in spite of its submesoscale size, affects the larger-scale flow through an eddy PV flux. During and after the ITEs formation, an eddy PV flux is induced that is directed upward along its isopycnal layer, towards the frontal outcrop. The eddy PV flux arises due to the tendency of frontal instabilities to subduct low-PV surface waters, while upwelling high-PV fluid from the pycnocline. This flux was found to scale with the wind-driven surface frictional PV flux. The relation between the eddy and frictional PV fluxes is nonlocal in time and space, as the eddy PV flux persists long after the wind-stress is turned off and is found in the interior of the fluid where frictional forces are weak. The effect of the ITE on the larger-scale flow is two-fold: it accelerates the along-front flow as well as induces a bolus velocity directed down the frontal outcrop with a magnitude that scales with the Ekman velocity at the time of wind-forcing.

In coarse-grid general circulation models that cannot resolve eddies, the effects of eddies on the larger-scale circulation must be parameterized. One of the key eddy-driven quantities that must be parameterized is the bolus velocity since it contributes to the advection of tracers in the ocean (Gent et al., 1995). Greatbatch (1998) has emphasized the equivalence between parameterizations for the bolus velocity and isopycnal eddy PV fluxes. Parameterizations for eddy 
PV fluxes invoke a down-gradient formulation with an eddy diffusivity that has a dependence on the properties of the flow (e.g. Greatbatch, 1998; Wardle and Marshall, 2000). The numerical experiment presented here suggests that submesoscale eddy PV fluxes at wind-forced upper-ocean baroclinic currents show a dependence not only on the baroclinicity of the flow but also on the atmospheric forcing through the wind-induced frictional PV flux. However, this dependence is nonlocal, and therefore, in order to parameterize the eddy PV flux and bolus velocity associated with wind-generated ITEs, a relation between the temporal variability of the eddy PV flux and wind-stress would have to be established. Understanding the fundamental physics behind this nonlocal relation and the development of an ITE PV flux parameterization will be the subject of future research.

Acknowledgments. This work was supported by NSF grants OCE-03-51191, OCE-05-49699, and OCE-0612058. Comments by Kurt Polzin, Mike Spall, and six anonymous reviewers were very helpful in improving the manuscript. 


\section{References}

Andrews, D. G., Holton, J., Leovy, C., 1987. Middle atmosphere dynamics. Academic Press.

Boccaletti, G., Ferrari, R., Fox-Kemper, B., 2007. Mixed layer instabilities and restratification. J. Phys. Oceanogr. 37, 2228-2250.

Capet, X., McWilliams, J., Molemaker, M., Shchepetkin, A., 2008. Mesoscale to submesoscale transition in the California Current system. Part II: Frontal processes. J. Phys. Oceanogr. 38, 44-64.

D'Asaro, E. A., 1988. Generation of submesoscale vorticies: a new mechanism. J. Geophys. Res. 93, 6685-6693.

Dugan, J. P., Mied, R., Mignerey, P., Schuetz, A., 1982. Compact, intrathermocline eddies in the Sargasso Sea. J. Geophys. Res. 87, 385-393.

Gent, P. R., Willebrand, J., McDougall, T. J., McWilliams, J. C., 1995. Parameterizing eddy-induced tracer transports in ocean circulation models. J. Phys. Oceanogr. 25, 463-474.

Gill, A. E., 1981. Homogeneous intrusions in a rotating stratified fluid. J. Fluid Mech. 103, 275-295.

Greatbatch, R. J., 1998. Exploring the relationship between eddy-induced transport velocity, vertical momentum transfer, and the isopycnal flux of potential vorticity. J. Phys. Oceanogr. 28, 422-432.

Haynes, P., McIntyre, M., 1987. On the evolution of vorticity and potential vorticity in the presence of diabatic heating and frictional or other forces. J. Atmos. Sci. 44.

Hedstrom, K., Armi, L., 1988. An experimental study of homogeneous lenses in a stratified rotating fluid. J. Fluid Mech. 191, 535-556.

Hoskins, B. J., McIntyre, M. E., Robertson, A. W., 1985. On the use and 
significance of isentropic potential vorticity maps. Qt. J. R. Met. Soc. 111, $877-946$.

Kostianoy, A. G., Belkin, I. M., 1989. A survey of observations on intrathermocline eddies in the world ocean. In: Nihoul, J. C. J., Jamart, B. M. (Eds.), Mesoscale/Synoptic Coherent Structures in Geophysical Turbulence. Elsevier, pp. 821-841.

Large, W. G., McWilliams, J. C., Doney, S. C., 1994. Oceanic vertical mixing: a review and a model with a nonlocal boundary layer parameterization. Rev. Geophys. 32, 363-403.

Marshall, J., Schott, F., 1999. Open ocean deep convection: observations, models and theory. Rev. Geophys. 37, 1-64.

Marshall, J. C., Nurser, A. J. G., 1992. Fluid dynamics of oceanic thermocline ventilation. J. Phys. Oceanogr. 22, 583-595.

McWilliams, J. C., 1985. Submesoscale, coherent vorticies in the ocean. Rev. Geophys. 23, 165-182.

Rhines, P. B., Holland, W. R., 1979. A theoretical discussion of eddy-driven mean flows. Dyn. Atmos. Oceans 3, 289-325.

Shchepetkin, A. F., McWilliams, J. C., 2005. The Regional Ocean Modeling System (ROMS): a split-explicit, free-surface, topography-following coordinate oceanic model. Ocean Modelling 9, 347-404.

Spall, M. A., 1995. Frontogenesis, subduction, and cross-front exchange at upper ocean fronts. J. Geophys. Res. 100, 2543-2557.

Thomas, L. N., 2005. Destruction of potential vorticity by winds. J. Phys. Oceanogr. 35.

Thomas, L. N., 2007. Dynamical constraints on the extreme low values of the potential vorticity in the ocean. In: Proceedings of the 15th 'Aha Huliko'a Hawaiian Winter Workshop. University of Hawaii. 
Thomas, L. N., Lee, C. M., 2005. Intensification of ocean fronts by down-front winds. J. Phys. Oceanogr. 35, 1086-1102.

Wardle, R., Marshall, J., 2000. Representation of eddies in primitive equation models by a PV flux. J. Phys. Oceanogr. 30, 2481-2503.

Wilson, C., Williams, R. G., 2003. Why are eddy fluxes of potential vorticity difficult to parameterize? J. Phys. Oceanogr. 34, 142-155. 


\section{List of Figures}

1 A control volume $\mathscr{V}$ (dashed lines) with side surfaces coincident with isopycnals (gray contours) of buoyancy $b_{+}$and $b_{-}$that bound a frontal zone for a front in the Northern Hemisphere. The "down-front" wind-stress $\tau_{w}$ and flux of buoyancy from the ocean to the atmosphere $\mathscr{B}_{\text {atm }}$ will result in an upward PV flux $\mathbf{J}$ at the sea-surface.

2 Initial conditions for the zonal velocity (shades) and density (white contours) of the numerical experiment. The contour interval for the density is $0.1 \mathrm{~kg} \mathrm{~m}^{-3}$. The vertical grid spacing is indicated on the left axis.

3 The density at $z=-3 \mathrm{~m}$. Time is indicated at the top of each panel in units of inertial periods and the 28.3, 28.5, and 28.7 $\mathrm{kg} \mathrm{m}^{-3}$ isopycnals are contoured in black, grey, and light grey, respectively.

4 The potential vorticity on the $28.335 \mathrm{~kg} \mathrm{~m}^{-3}$ isopycnal surface. Time is indicated at the top of each panel in units of inertial periods. Also plotted in black dots are the locations of Lagrangian floats released in the low-PV plume. Light shades indicate low PV values.

5 Zonal velocity (shades) and density (white contours) on a meridional section that strikes through the center of the ITE at $x=27.5 \mathrm{~km}, y=65 \mathrm{~km}$, and $t=10$ inertial periods.

6 Radial profiles of properties of the velocity field of the ITE at $z=-80.9 \mathrm{~m}$ and at $t=9.9$ inertial periods: $(a)$ the magnitude of the azimuthal velocity, $(b)$ the vertical component of the relative vorticity normalized by $f$, and $(c)$ the centrifugal force, i.e. the left hand side of (21) (black circles) and the right hand side of (21)(gray dots).

7 Top panel: the PV integrated over a control volume $\mathscr{V}$ bounded by the 28.3 and $28.5 \mathrm{~kg} \mathrm{~m}^{-3}$ isopycnal surfaces. Bottom panel: time integrals of terms in the PV budget (22), see text for details.

8 Left panel: the frictional PV flux integrated over the outcrop area at $z=-3 \mathrm{~m}$ of various isopycnal layers, $\mathscr{J}_{z}^{F}$, versus the central density of each layer. Right panel: the time integral of minus this area integral, $-\int_{s=0}^{s=t} \mathscr{J}_{z}^{F} \mathrm{~d} s$. 
9 Constituents of the potential vorticity (top), terms in the equations for the vertical vorticity (middle) and north-south buoyancy gradient (bottom) averaged over Lagrangian particles released in the low-PV plume shown in figure 4. See text for notation.

10 Terms in the averaged isopycnal zonal momentum equation (35) on the $28.3-28.5 \mathrm{~kg} \mathrm{~m}^{-3}$ isopycnal layer: $(a) \partial \overline{u_{\rho}} / \partial t,(b)$ $-\hat{q}_{\rho} \bar{z}_{\rho} \hat{v}_{\rho},(c) \overline{z_{\rho} q_{\rho}^{\prime \prime} v_{\rho}^{\prime \prime}}$, and $(d) \overline{X_{\rho}}$. The location of the center of the ITE (white line) is also plotted in $(a)$.

11 Relation of the eddy PV flux to the structure of the ITE and the averaged PV: $(a) \hat{q_{\rho}} \Delta b,(b) \overline{z_{\rho} q_{\rho}^{\prime \prime} v_{\rho}^{\prime \prime}} \Delta b L_{x}$, and $(c)$ the deviation from the thickness averaged $\mathrm{PV}, q_{\rho}^{\prime \prime} \Delta b$, (shades) and the meridional velocity, $v_{\rho}^{\prime \prime}$, (contours) on the $28.3-28.5 \mathrm{~kg} \mathrm{~m}^{-3}$ isopycnal layer at $t=8.2$ inertial periods. The contour interval in $(c)$ is $0.05 \mathrm{~m} \mathrm{~s}^{-1}$, positive (negative) values are contoured with solid (dashed) lines, and the zero contour is indicated by the heavy line.

12 Hovmöller diagrams of $(a)$ the thickness-weighted, isopycnally averaged PV (multiplied by $\Delta b$ ) and $(b)$ the eddy induced isopycnal flux of PV (multiplied by $\Delta b L_{x}$ ) on the layer bounded by the 28.3 and $28.5 \mathrm{~kg} \mathrm{~m}^{-3}$ isopycnal surfaces. The location of the maximum eddy PV flux (white dots) is also plotted in $(a)$. (c) Timeseries of the integrated maximum eddy PV flux, $\left.\mathscr{J}_{y}^{e}\right|_{y=y_{\max }}$, (black) and the frictional PV flux integrated over the outcrop area of the $28.3-28.5 \mathrm{~kg}$ $\mathrm{m}^{-3}$ isopycnal layer, $\mathscr{J}_{z}^{F}$, (gray) along with the scaling $\left[\mathscr{J}_{z}^{F}\right]=\tau_{w}^{x} \Delta b L_{x} /\left(\rho_{o} \delta_{e}\right)$ (dashed).

13 Hovmöller diagrams of $(a)$ the thickness-weighted, isopycnally averaged meridional velocity, $\hat{v}_{\rho},(b)$ the zonally averaged meridional velocity $\overline{v_{\rho}},(c)$ the bolus velocity $v_{\rho}^{*}$, and $(d)$ $-\overline{z_{\rho} q_{\rho}^{\prime \prime} v_{\rho}^{\prime \prime}} / f$ on the $28.3-28.5 \mathrm{~kg} \mathrm{~m}^{-3}$ isopycnal layer. The location of the center of the ITE (magenta line) is also plotted in $(a)$. 


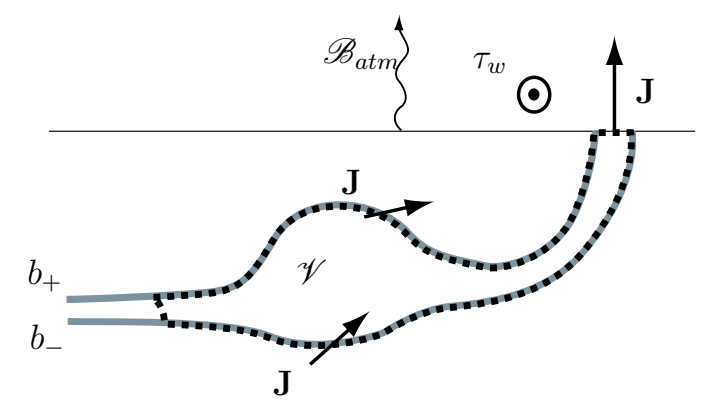

Fig. 1. A control volume $\mathscr{V}$ (dashed lines) with side surfaces coincident with isopycnals (gray contours) of buoyancy $b_{+}$and $b_{-}$that bound a frontal zone for a front in the Northern Hemisphere. The "down-front" wind-stress $\tau_{w}$ and flux of buoyancy from the ocean to the atmosphere $\mathscr{B}_{\text {atm }}$ will result in an upward PV flux $\mathbf{J}$ at the sea-surface. 


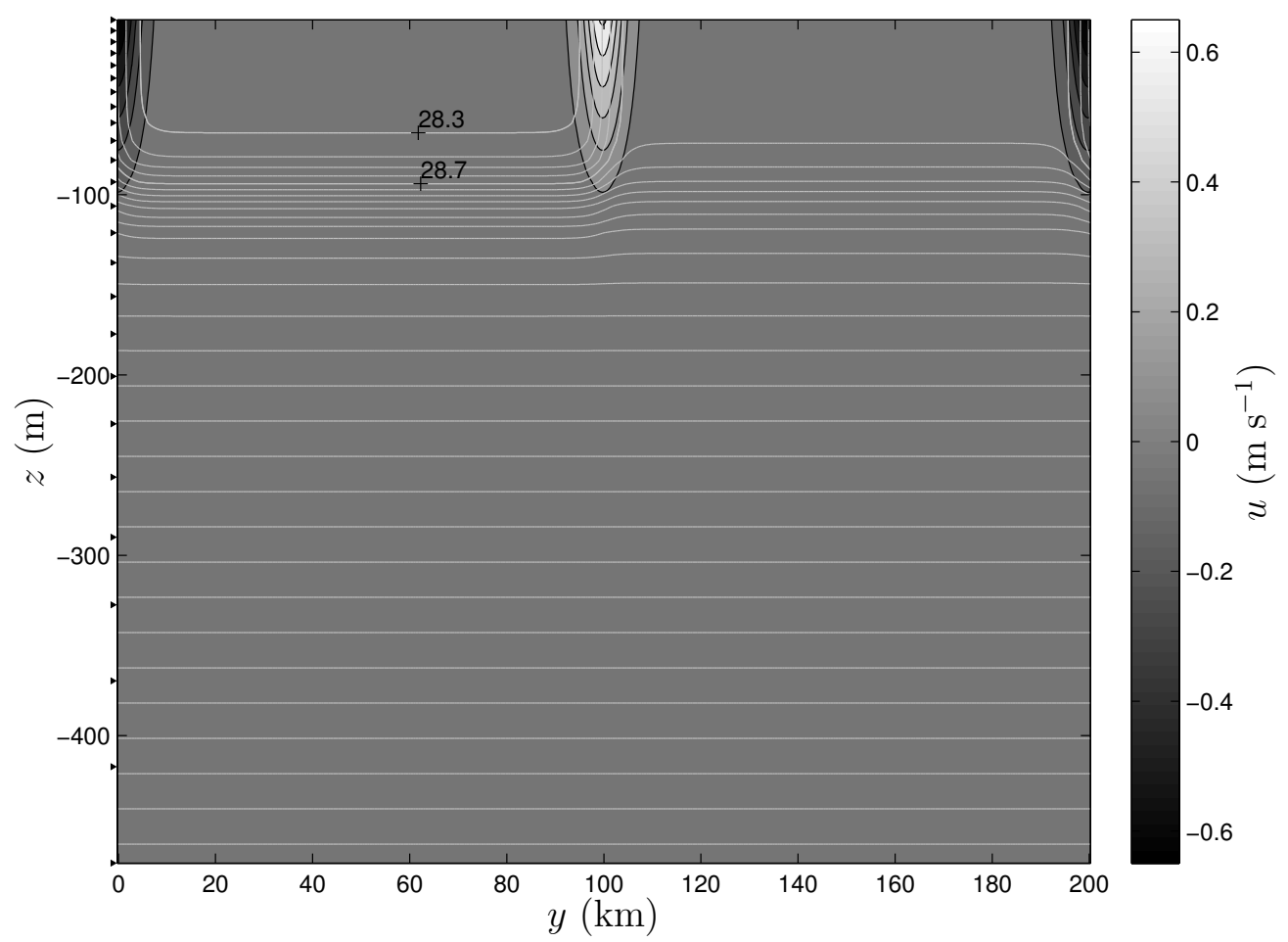

Fig. 2. Initial conditions for the zonal velocity (shades) and density (white contours) of the numerical experiment. The contour interval for the density is $0.1 \mathrm{~kg} \mathrm{~m}^{-3}$. The vertical grid spacing is indicated on the left axis. 

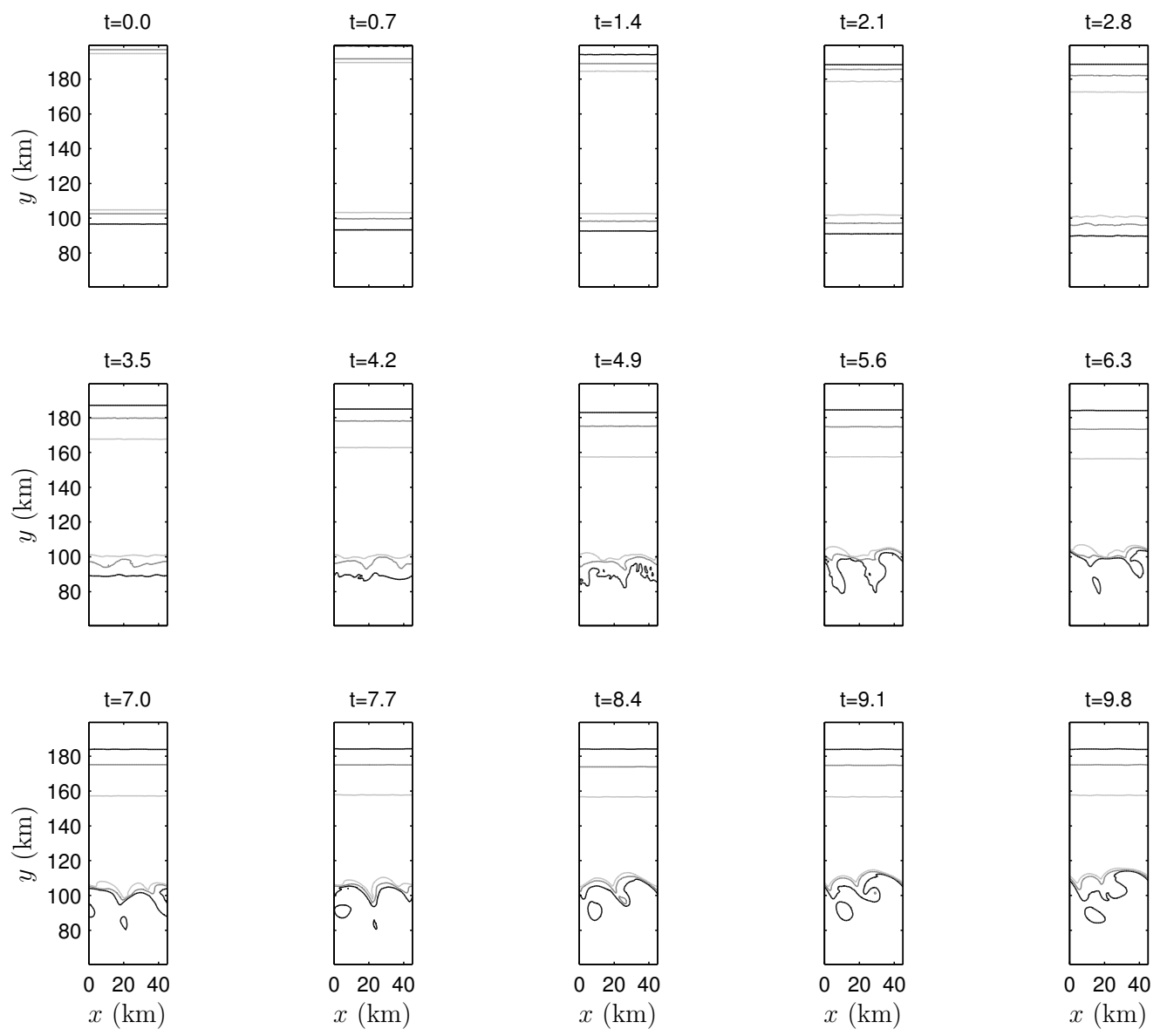

Fig. 3. The density at $z=-3 \mathrm{~m}$. Time is indicated at the top of each panel in units of inertial periods and the $28.3,28.5$, and $28.7 \mathrm{~kg} \mathrm{~m}^{-3}$ isopycnals are contoured in black, grey, and light grey, respectively. 


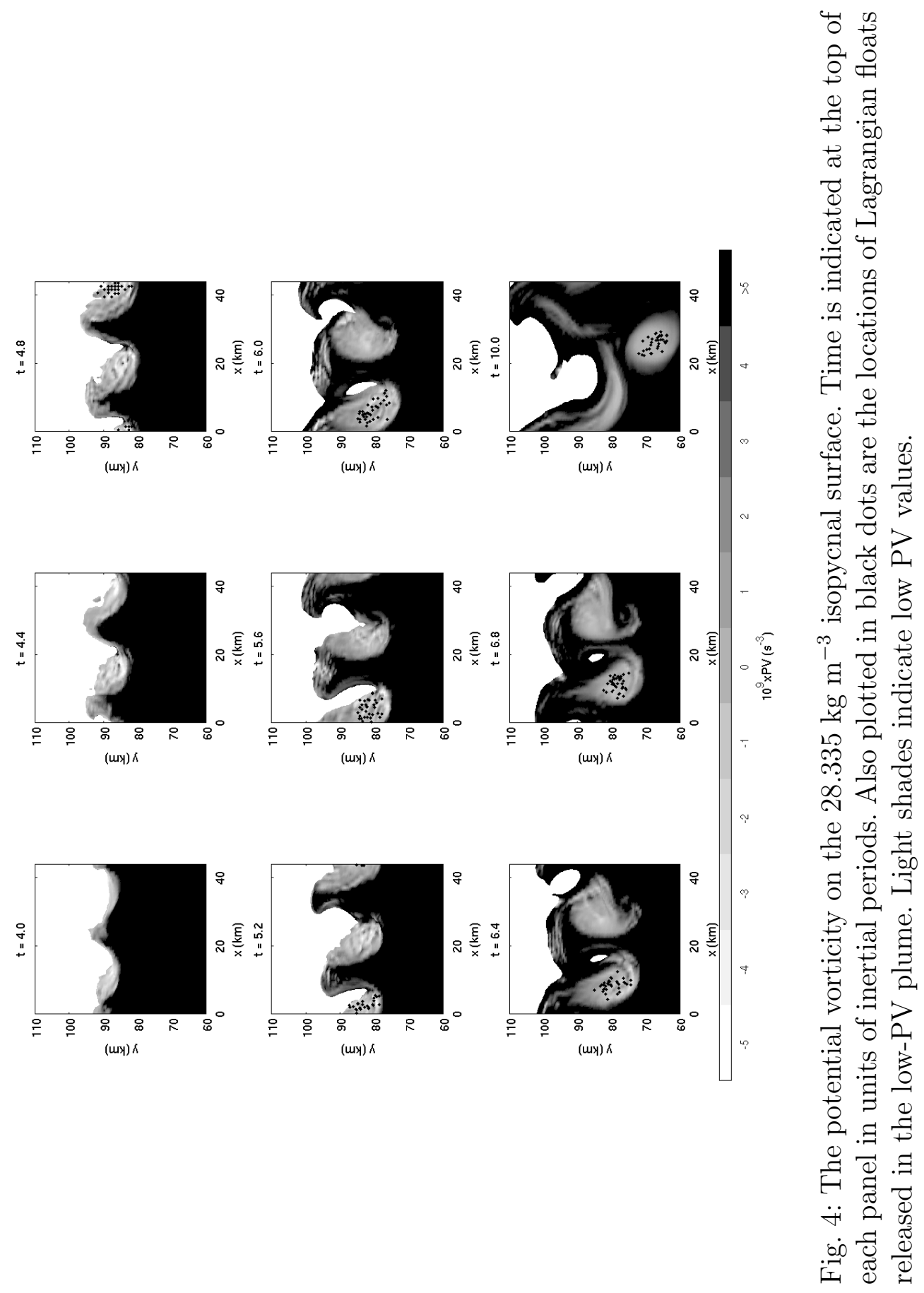




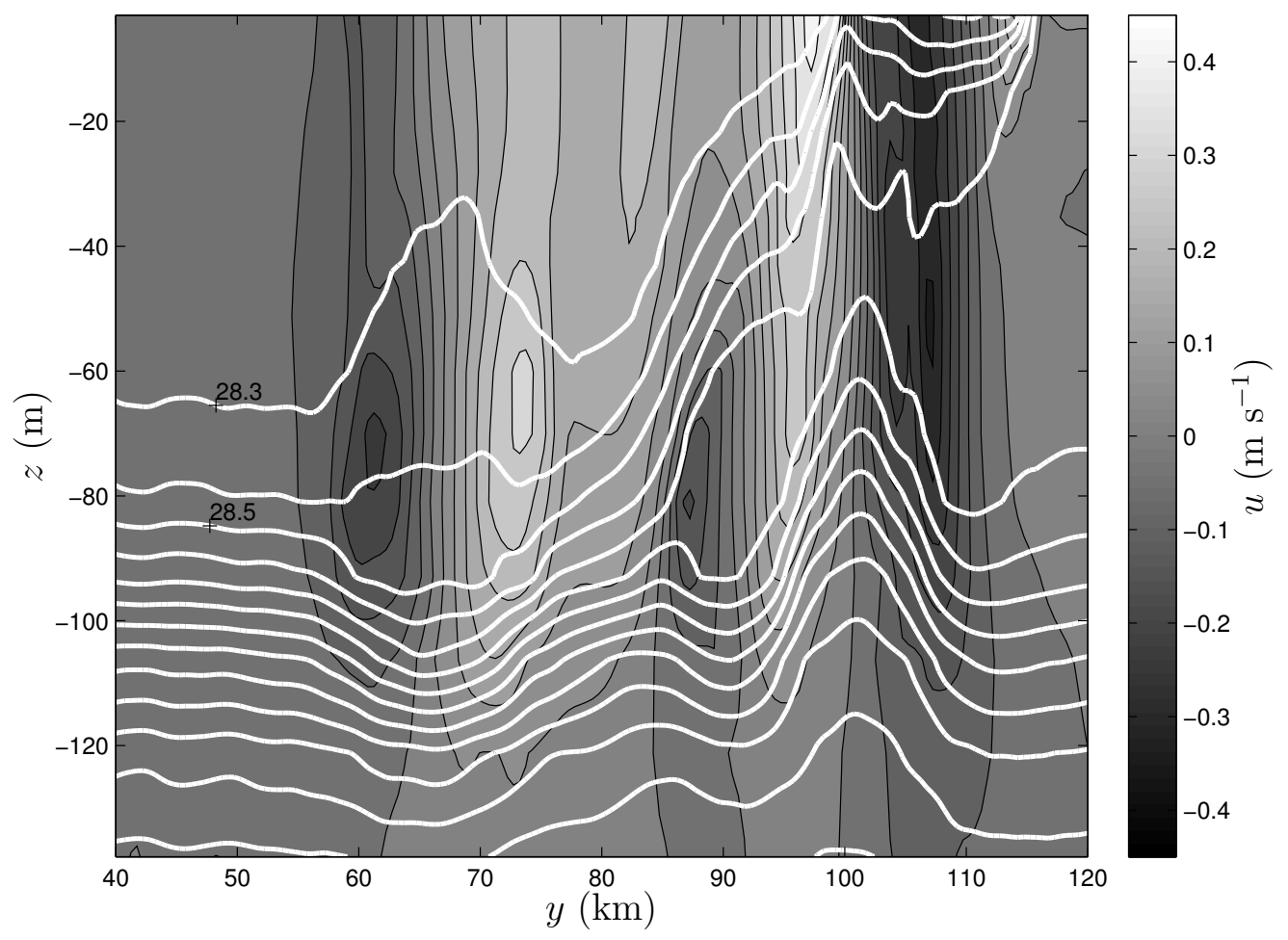

Fig. 5. Zonal velocity (shades) and density (white contours) on a meridional section that strikes through the center of the ITE at $x=27.5 \mathrm{~km}, y=65 \mathrm{~km}$, and $t=10$ inertial periods. 

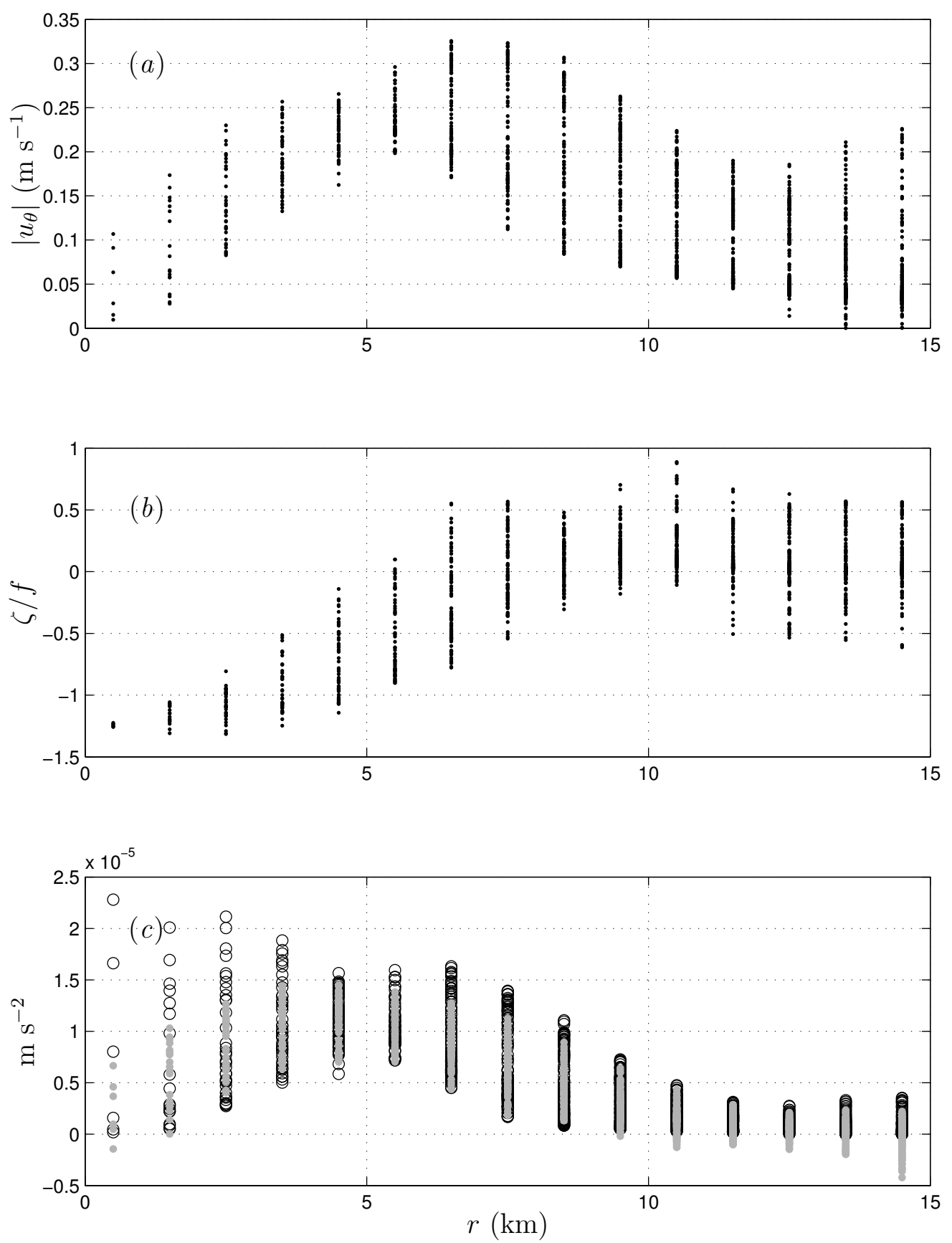

Fig. 6. Radial profiles of properties of the velocity field of the ITE at $z=-80.9$ $\mathrm{m}$ and at $t=9.9$ inertial periods: $(a)$ the magnitude of the azimuthal velocity, (b) the vertical component of the relative vorticity normalized by $f$, and $(c)$ the centrifugal force, i.e. the left hand side of (21) (black circles) and the right hand side of (21)(gray dots). 

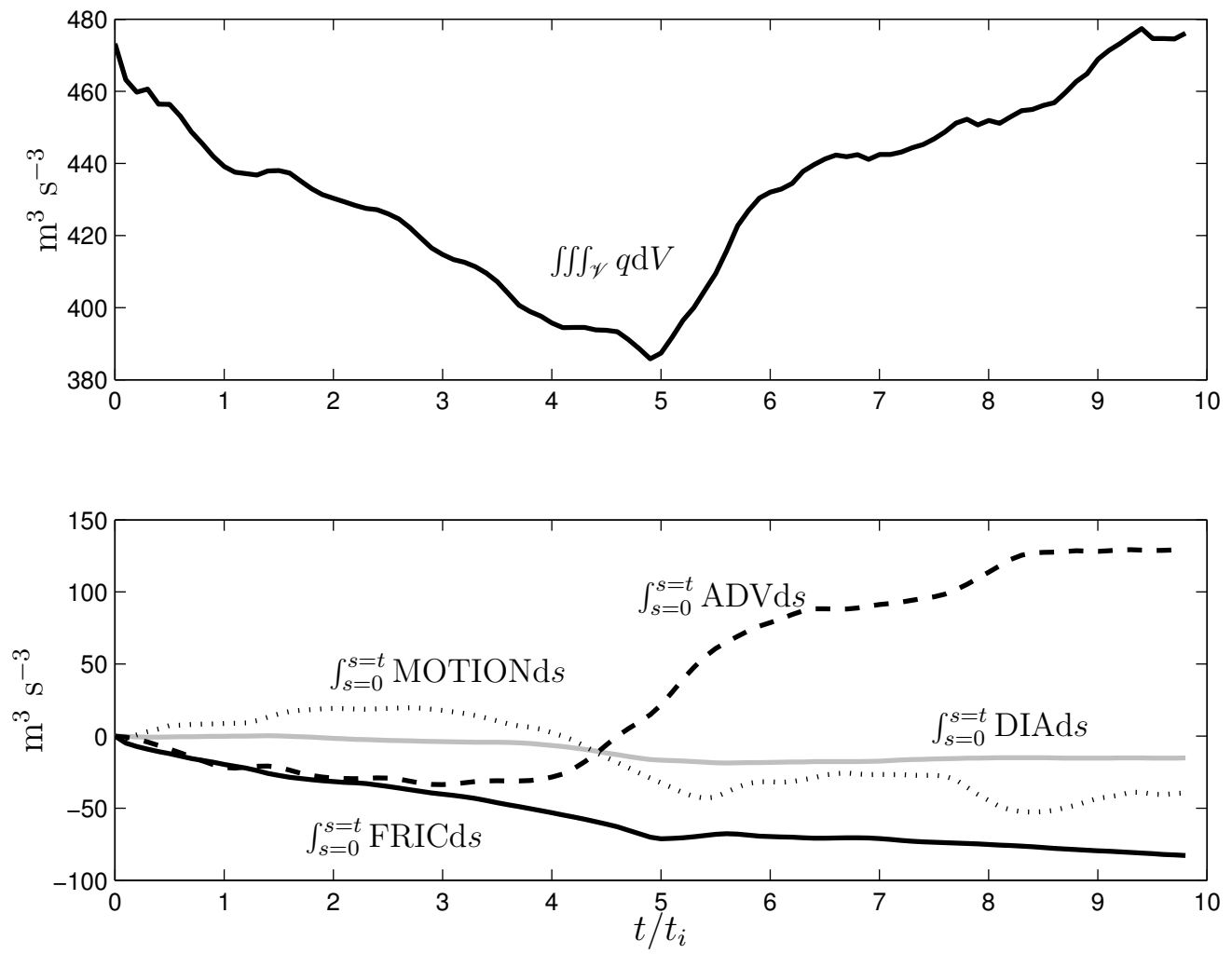

Fig. 7. Top panel: the PV integrated over a control volume $\mathscr{V}$ bounded by the 28.3 and $28.5 \mathrm{~kg} \mathrm{~m}^{-3}$ isopycnal surfaces. Bottom panel: time integrals of terms in the PV budget (22), see text for details. 

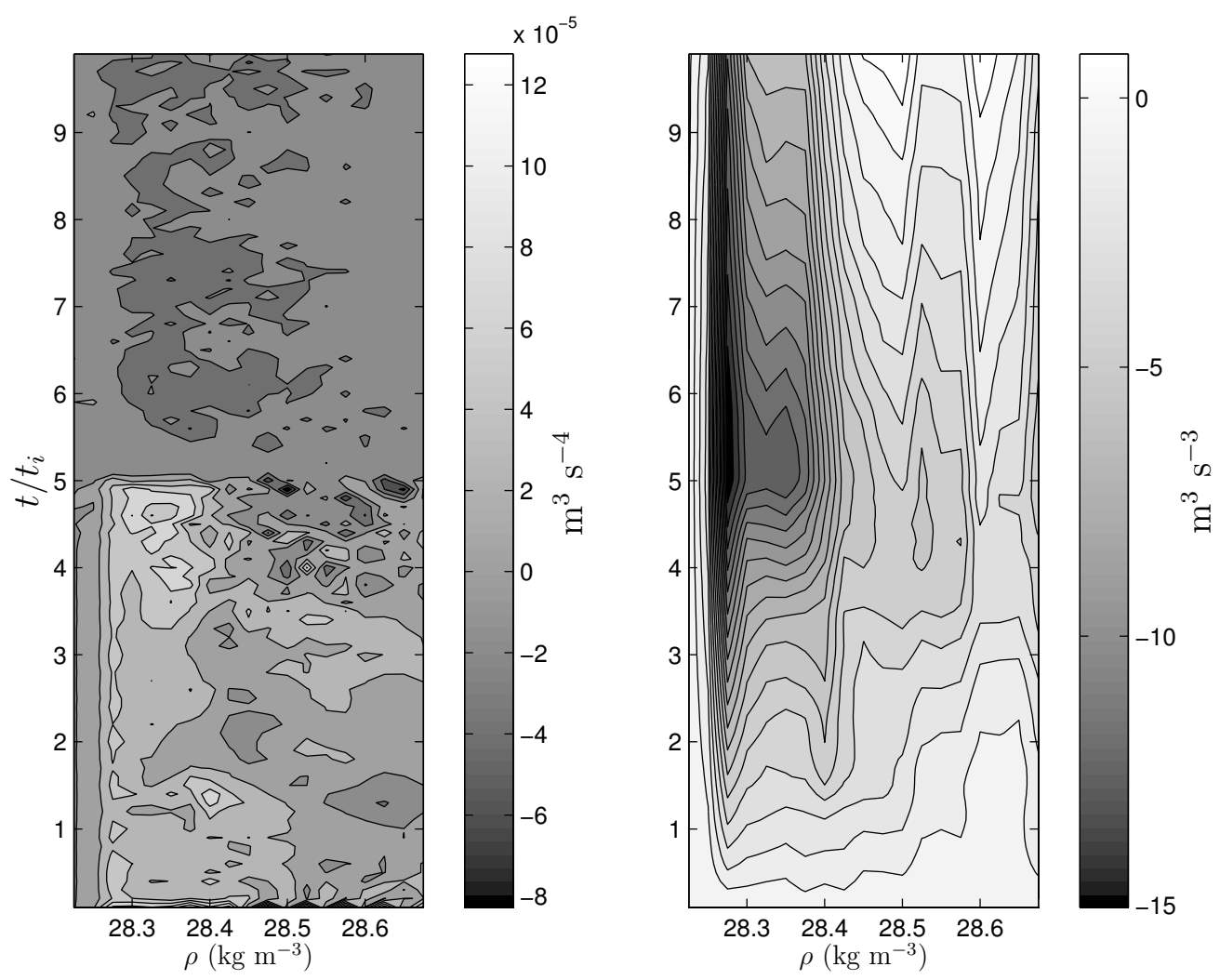

Fig. 8. Left panel: the frictional PV flux integrated over the outcrop area at $z=-3$ $\mathrm{m}$ of various isopycnal layers, $\mathscr{J}_{z}^{F}$, versus the central density of each layer. Right panel: the time integral of minus this area integral, $-\int_{s=0}^{s=t} \mathscr{J}_{z}^{F} \mathrm{~d} s$. 

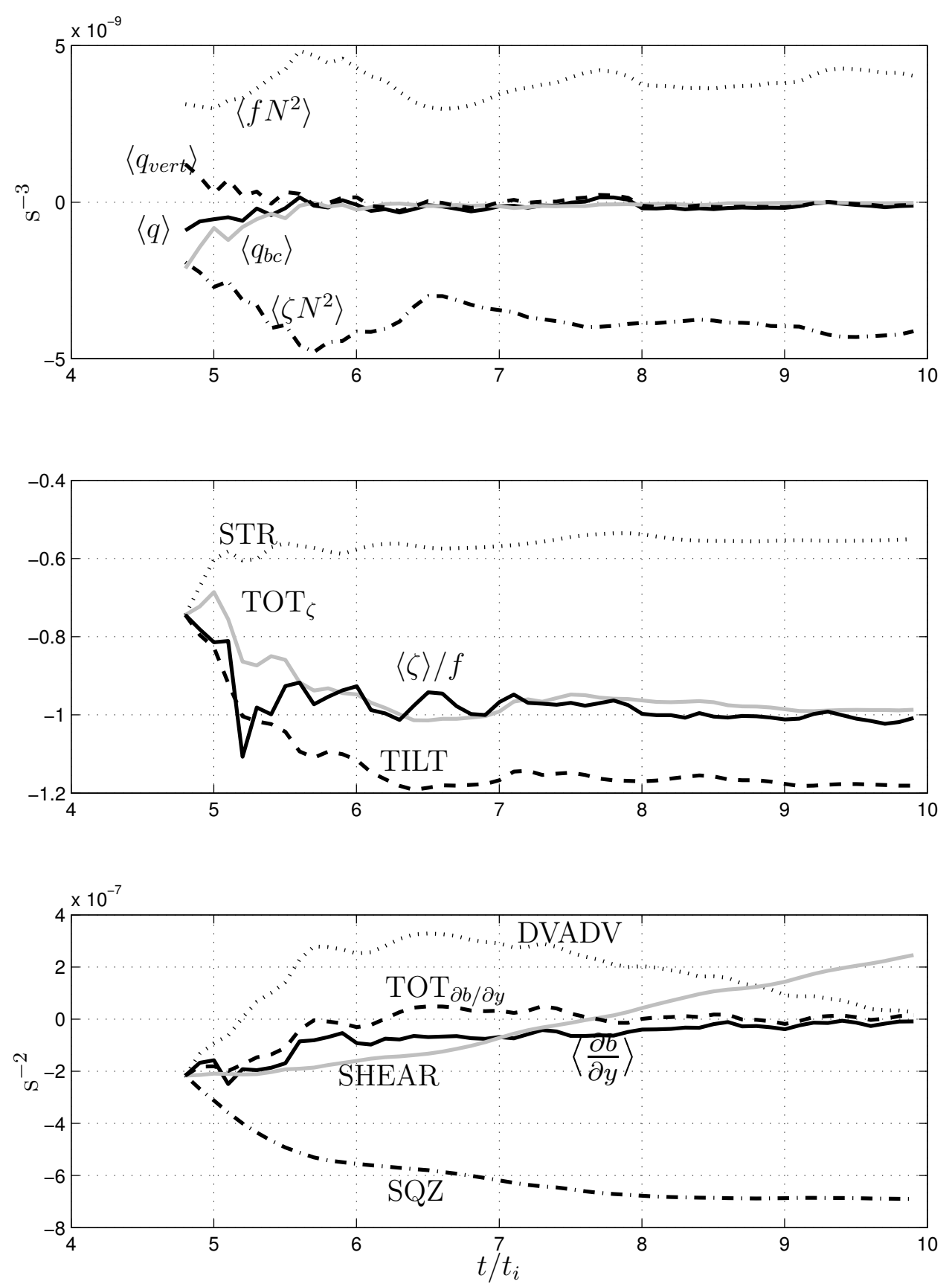

Fig. 9. Constituents of the potential vorticity (top), terms in the equations for the vertical vorticity (middle) and north-south buoyancy gradient (bottom) averaged over Lagrangian particles released in the low-PV plume shown in figure 4. See text for notation. 

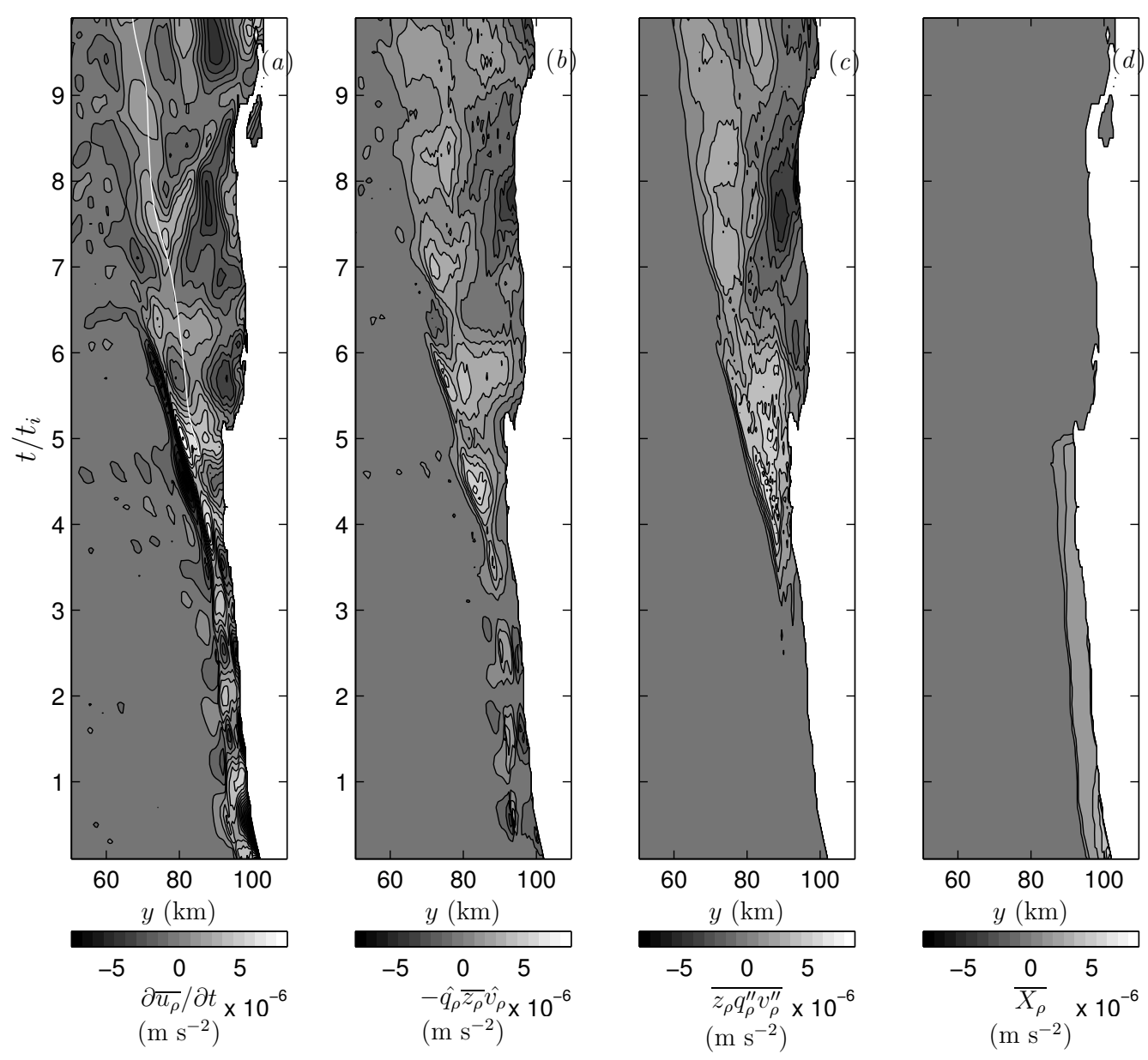

Fig. 10. Terms in the averaged isopycnal zonal momentum equation (35) on the 28.3-28.5 $\mathrm{kg} \mathrm{m}^{-3}$ isopycnal layer: $(a) \partial \overline{u_{\rho}} / \partial t,(b)-\hat{q}_{\rho} \bar{z}_{\rho} \hat{v}_{\rho},(c) \overline{z_{\rho} q_{\rho}^{\prime \prime} v_{\rho}^{\prime \prime}}$, and $(d) \overline{X_{\rho}}$. The location of the center of the ITE (white line) is also plotted in $(a)$. 

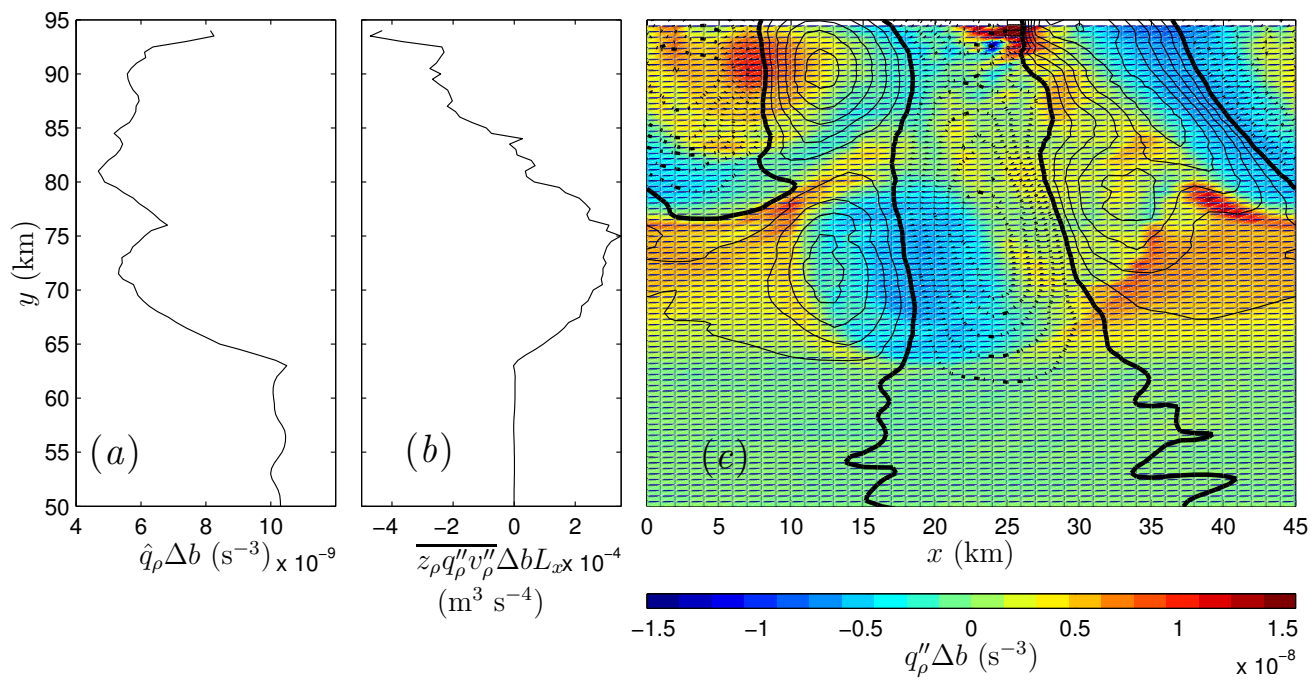

Fig. 11. Relation of the eddy PV flux to the structure of the ITE and the averaged $\mathrm{PV}:(a) \hat{q_{\rho}} \Delta b,(b) \overline{z_{\rho} q_{\rho}^{\prime \prime} v_{\rho}^{\prime \prime}} \Delta b L_{x}$, and $(c)$ the deviation from the thickness averaged $\mathrm{PV}, q_{\rho}^{\prime \prime} \Delta b$, (shades) and the meridional velocity, $v_{\rho}^{\prime \prime}$, (contours) on the $28.3-28.5 \mathrm{~kg}$ $\mathrm{m}^{-3}$ isopycnal layer at $t=8.2$ inertial periods. The contour interval in $(c)$ is 0.05 $\mathrm{m} \mathrm{s}^{-1}$, positive (negative) values are contoured with solid (dashed) lines, and the zero contour is indicated by the heavy line. 

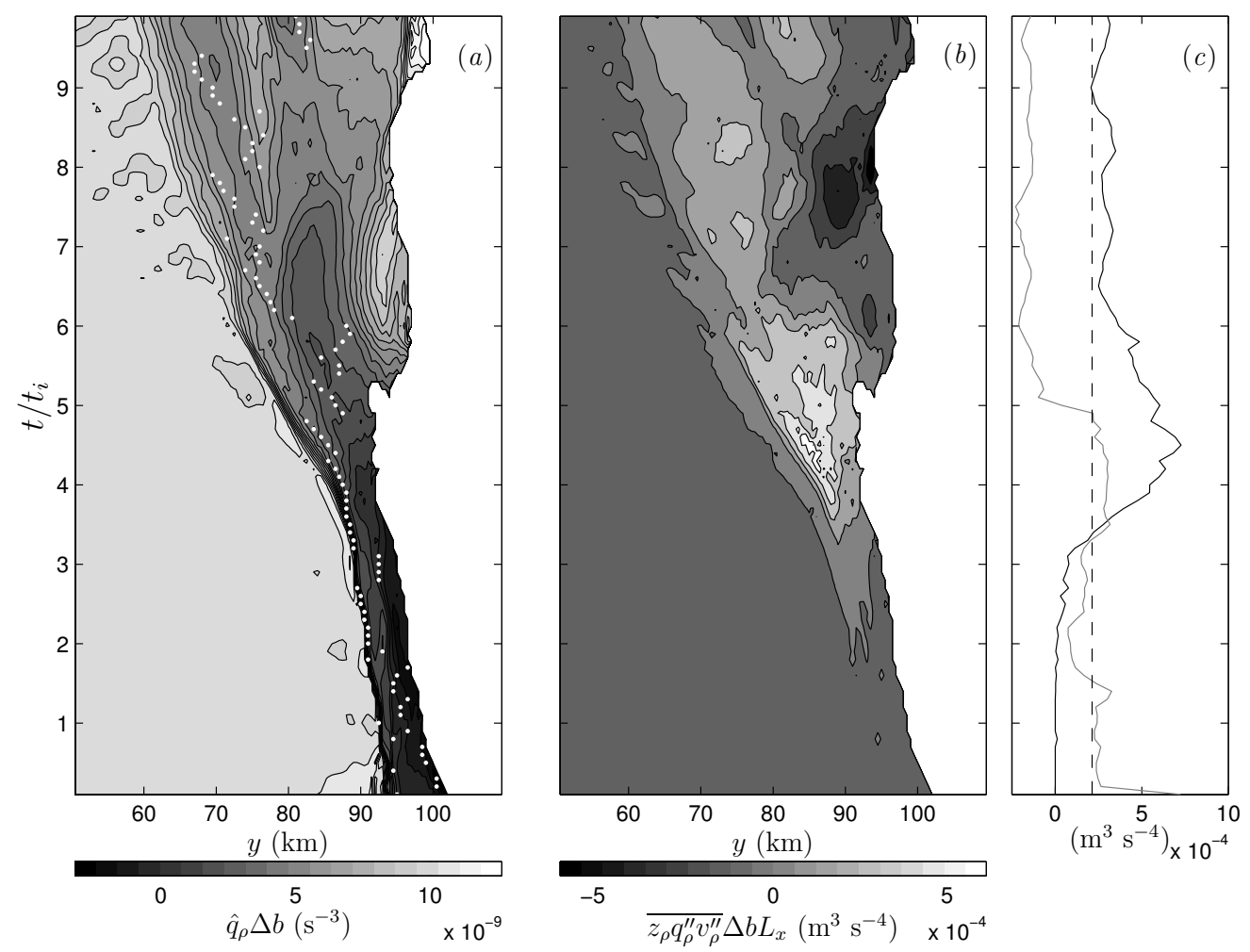

Fig. 12. Hovmöller diagrams of $(a)$ the thickness-weighted, isopycnally averaged $\mathrm{PV}$ (multiplied by $\Delta b$ ) and (b) the eddy induced isopycnal flux of PV (multiplied by $\Delta b L_{x}$ ) on the layer bounded by the 28.3 and $28.5 \mathrm{~kg} \mathrm{~m}^{-3}$ isopycnal surfaces. The location of the maximum eddy PV flux (white dots) is also plotted in $(a)$. (c) Timeseries of the integrated maximum eddy PV flux, $\left.\mathscr{J}_{y}^{e}\right|_{y=y_{\max }}$, (black) and the frictional PV flux integrated over the outcrop area of the $28.3-28.5 \mathrm{~kg} \mathrm{~m}^{-3}$ isopycnal layer, $\mathscr{J}_{z}^{F}$, (gray) along with the scaling $\left[\mathscr{J}_{z}^{F}\right]=\tau_{w}^{x} \Delta b L_{x} /\left(\rho_{o} \delta_{e}\right)$ (dashed). 

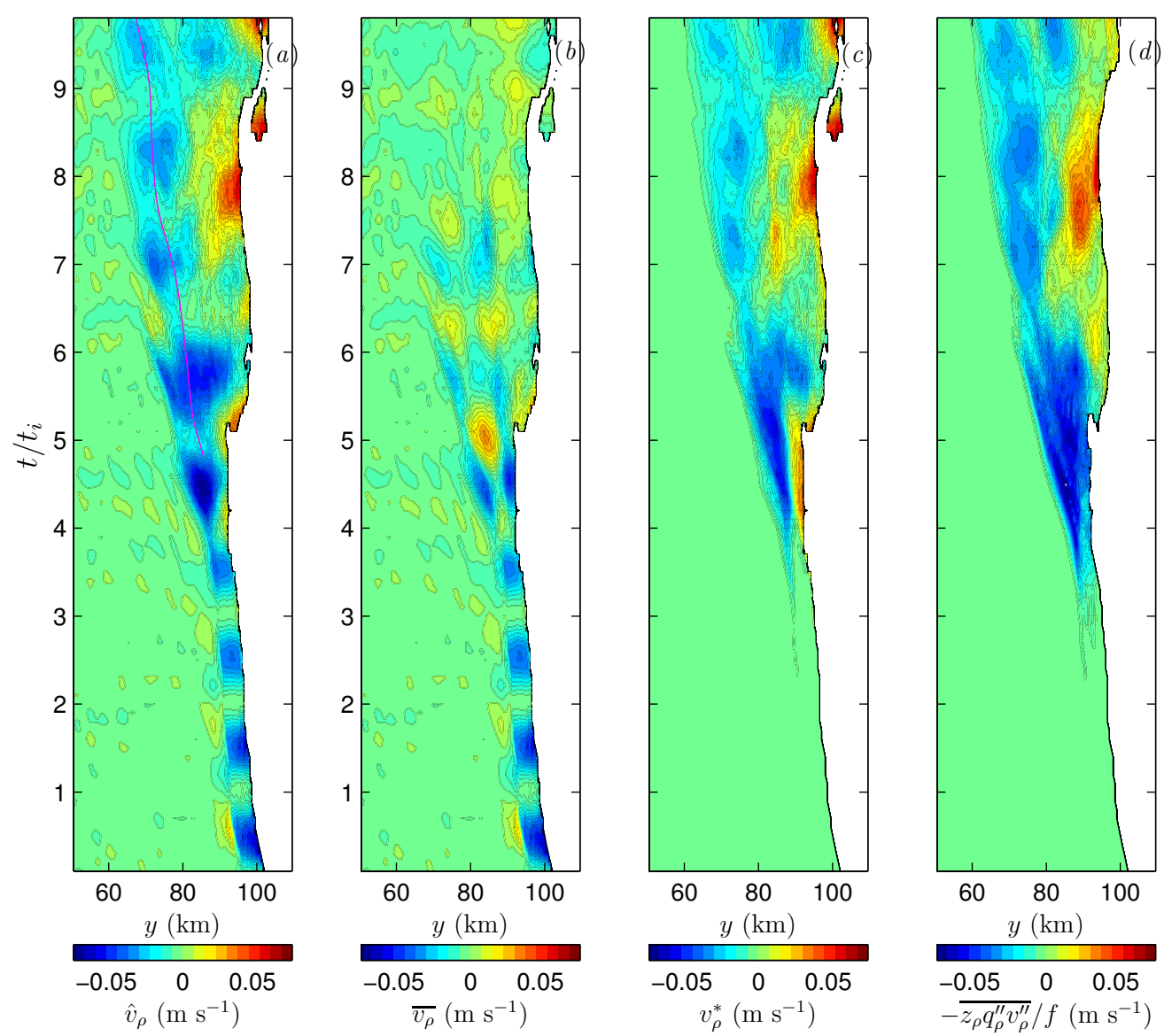

Fig. 13. Hovmöller diagrams of $(a)$ the thickness-weighted, isopycnally averaged meridional velocity, $\hat{v}_{\rho},(b)$ the zonally averaged meridional velocity $\overline{v_{\rho}},(c)$ the bolus velocity $v_{\rho}^{*}$, and $(d)-\overline{z_{\rho} q_{\rho}^{\prime \prime} v_{\rho}^{\prime \prime}} / f$ on the $28.3-28.5 \mathrm{~kg} \mathrm{~m}^{-3}$ isopycnal layer. The location of the center of the ITE (magenta line) is also plotted in $(a)$. 